\title{
Full Rescue of F508del-CFTR Processing and Function by CFTR Modulators Can Be Achieved by Removal of Two Regulatory Regions
}

\author{
Inna Uliyakina ${ }^{\mathbb{D}}$, Hugo M. Botelho ${ }^{\mathbb{D}}$, Ana C. da Paula, Sara Afonso, Miguel J. Lobo, \\ Verónica Felício ${ }^{(D)}$, Carlos M. Farinha ${ }^{(D)}$ and Margarida D. Amaral *(D) \\ BioISI-Biosystems \& Integrative Sciences Institute, Faculty of Sciences, University of Lisboa, \\ 1749-016 Lisboa, Portugal; iiuliyakina@fc.ul.pt (I.U.); hmbotelho@fc.ul.pt (H.M.B.); \\ acdapaula@fc.ul.pt (A.C.d.P.); scafonso@fc.ul.pt (S.A.); mglobo@fc.ul.pt (M.J.L.); vmfelicio@fc.ul.pt (V.F.); \\ cmfarinha@fc.ul.pt (C.M.F.) \\ * Correspondence: mdamaral@fc.ul.pt; Tel.: +351-217500861
}

Received: 22 May 2020; Accepted: 22 June 2020; Published: 25 June 2020

\begin{abstract}
Cystic Fibrosis (CF) is caused by mutations in the CF Transmembrane conductance Regulator (CFTR), the only ATP-binding cassette (ABC) transporter functioning as a channel. Unique to CFTR is a regulatory domain which includes a highly conformationally dynamic region-the regulatory extension (RE). The first nucleotide-binding domain of CFTR contains another dynamic region-regulatory insertion (RI). Removal of RI rescues the trafficking defect of CFTR with F508del, the most common CF-causing mutation. Here we aimed to assess the impact of RE removal (with/without RI or genetic revertants) on F508del-CFTR trafficking and how CFTR modulator drugs VX-809/lumacaftor and VX-770/ivacaftor rescue these variants. We generated cell lines expressing $\triangle$ RE and $\triangle$ RI CFTR (with/without genetic revertants) and assessed CFTR expression, stability, plasma membrane levels, and channel activity. Our data demonstrated that $\Delta$ RI significantly enhanced rescue of F508del-CFTR by VX-809. While the presence of the RI seems to be precluding full rescue of F508del-CFTR processing by VX-809, this region appears essential to rescue its function by VX-770, suggesting some contradictory role in rescue of F508del-CFTR by these two modulators. This negative impact of RI removal on VX-770-stimulated currents on F508del-CFTR can be compensated by deletion of the RE which also leads to the stabilization of this mutant. Despite both regions being conformationally dynamic, RI precludes F508del-CFTR processing while RE affects mostly its stability and channel opening.
\end{abstract}

Keywords: $\mathrm{ABC}$ transporters; drug action; regulatory extension; regulatory insertion; mechanism of action

\section{Introduction}

Cystic Fibrosis (CF), a life-threatening recessive disorder affecting 80,000 individuals worldwide, is caused by mutations in the gene encoding the CF transmembrane conductance regulator (CFTR) protein present at the apical membrane of epithelial cells. This is the only member of the ATP-binding cassette $(\mathrm{ABC})$ transporter family functioning as a channel, more precisely a cyclic Adenosine Monophosphate (cAMP)-dependent chloride $\left(\mathrm{Cl}^{-}\right) /$bicarbonate $\left(\mathrm{HCO}_{3}{ }^{-}\right)$channel. It consists of two membrane-spanning domains (MSD1/2), two nucleotide binding domains (NBD1/2) and a cytoplasmic regulatory domain (RD), which is unique to CFTR [1]. The MSDs are linked via intra- and extra-cellular loops (ICLs, ECLs, respectively). ATP binding promoting NBD1:NBD2 dimerization preceded by phosphorylation of RD at multiple sites lead to channel gating [2]. The NBD1:NBD2 and ICL4:NBD1 
interfaces were shown to represent critical folding conformational sites [3-5] important for the gating and maturation of the CFTR protein $[5,6]$.

Although, >2000 CFTR gene mutations were reported (http://www.genet.sickkids.on.ca), one mutation-F508del-occurs in $85 \%$ of CF patients. This NBD1 mutant fails to traffic to the plasma membrane (PM) due to protein misfolding and retention by the endoplasmic reticulum quality control that targets it for premature proteasomal degradation. F508del-CFTR folding is a complex and inefficient process but it can be rescued, at least partially, by several treatments. These include low temperature incubation [7], genetic revertants [4,5,8-13] or pharmacological agents, like "corrector" VX-809 (lumacaftor) [14], one of the first CFTR modulator drugs to receive approval from the United States Food and Drug Administration in combination with potentiator VX-770/ivacaftor. Determining the additive/ synergistic rescue of F508del-CFTR by small molecule correctors together with other rescuing agents/revertants is very valuable to determine the mechanism of action of these CFTR modulators [5].

Comparative studies of CFTR with other ABC transporters are very powerful to understand the uniqueness of some of its regions, their influence on CFTR maturation and function as well as how they affect distinctive binding of CFTR to modulator drugs. Two such unique regions are present in NBD1 of CFTR, which are absent in NBDs of other ABC transporters (Figure S1) - the regulatory extension (RE) and regulatory insertion (RI). Both regions were described as highly conformationally dynamic $[3,15]$ following PKA phosphorylation at certain residues $\left({ }^{660} \mathrm{Ser},{ }^{670} \mathrm{Ser}\right.$, and $\left.{ }^{422} \mathrm{Ser}\right)[16,17]$. Importantly, removal of the 32-amino acid RI $(\Delta R I)$ was reported to rescue traffic of F508del-CFTR [13].

Our first goal was to assess the impact of removing the 30 amino acid RE $(\triangle R E)$ alone or jointly with $\triangle \mathrm{RI}$ on F508del-CFTR trafficking. Because there is some controversy regarding the RI and RE boundaries $[3,13,15,18-20]$ which have structural implications, we tested the short and long versions of both regions (Figure S1). Secondly, we aimed to evaluate how RE and or RI removal from F508del-CFTR influences the rescue of this mutant by genetic revertants. Our third and final goal was to determine how the traffic and function of these combined variants of F508del-CFTR ( $\triangle R E, \triangle R I$ plus genetic revertants) are rescued by CFTR modulator drugs VX-809 (corrector) and VX-770 (potentiator) to gain further insight into their mechanism of action.

Our data show that although F508del-CFTR without RE did not traffic to the PM, it showed a dramatic stabilization of its immature form (evidencing a turnover rate $\sim 2 \times$ lower than that of wt-CFTR. Results also show that, while $\Delta$ RI further increased processing of F508del-CFTR with revertants to almost wt-CFTR levels, RE removal completely abolished their processing, thus highlighting the different impact of the two dynamic regions on revertant-rescued F508del-CFTR. Most strikingly, although VX-809 rescued $\Delta$ RI-F508del-CFTR and $\Delta$ RE- $\triangle$ RI-F508del-CFTR processing to wt-CFTR levels, to achieve maximal function of F508del-CFTR, removal of just RI was insufficient, as both RI and RE had to be absent from F508del-CFTR. These data indicate that removal of these two regions has a positive effect on the rescuing efficacy of F508del-CFTR by CFTR modulators.

\section{Results}

2.1. Removal of Short Regulatory Extension $\left(R E_{S}\right)$ Alone or with Regulatory Insertion (RI) has No Impact on 508del-CFTR Processing

Given the controversy in defining both RI and RE, we chose to generate two versions of these regions because of the respective structural implications. Indeed, $\mathrm{RI}_{\mathrm{L}}$ and $\mathrm{RE}_{\mathrm{L}}$ are the complete regions which are absent in other ABC transporters (Figure S1), whereas, their shorter versions appeared as structurally meaningful to be removed: $\mathrm{RI}_{\mathrm{S}}$ is strictly the region described as destructured in the crystal structure and $R E_{S}$ is the core of RE, i.e., just the $\beta$-strand [19]. The impact of deleting $R E_{S}$ - short $\operatorname{RE}\left(\Delta^{654}\right.$ Ser-Gly ${ }^{673}$, Figure S1) - was first assessed, either alone or together with RI in its short and long variants $\left(\Delta \mathrm{RI}_{\mathrm{S}}\right.$ and $\left.\Delta \mathrm{RI}_{\mathrm{L}}\right)$, on the in vivo processing of wt- and F508del-CFTR by Western blot (WB) of Baby Hamster Kidney (BHK) cells stably expressing such variants (Figure 1B,E,F and Table 1). Processing of CFTR was assessed by WB in terms of its fully-glycosylated form (also termed band C) 
corresponding to post-Golgi forms, assumedly at the PM. Unprocessed CFTR, in turn corresponds to its core-glycosylated, ER-specific form (also termed band B). Removal of $\mathrm{RE}_{\mathrm{S}}$ had no impact on processing of either F508del-CFTR or wt-CFTR (Figure 1B, lanes 5,10; Table 1), despite that the immature form of $\triangle \mathrm{RE}_{\mathrm{S}}$-F508del-CFTR appeared consistently at increased levels vs. those of F508del-CFTR (Figure 1B, lanes 5,2).

A
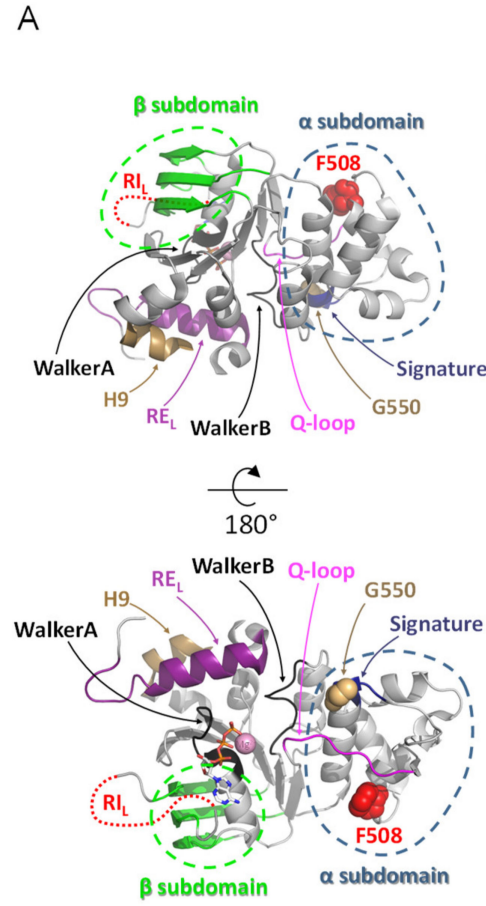
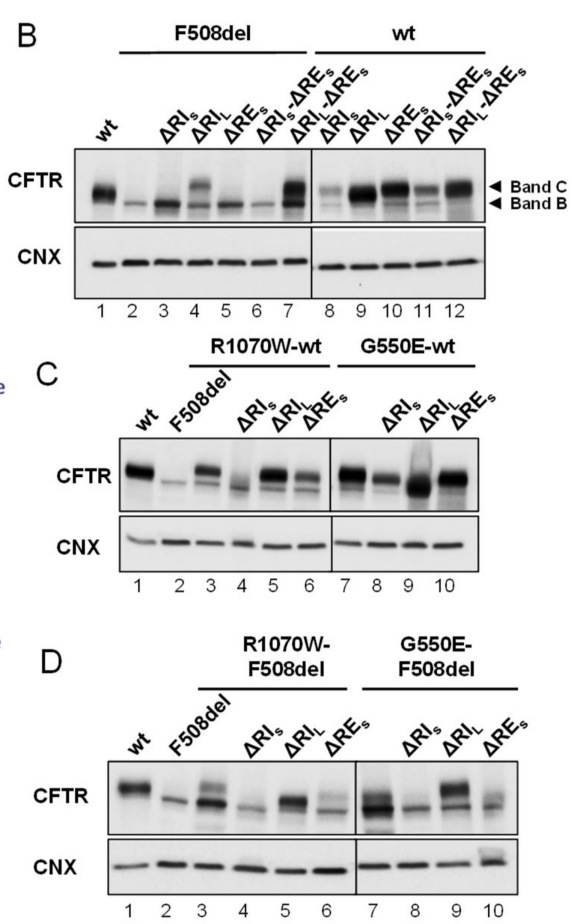
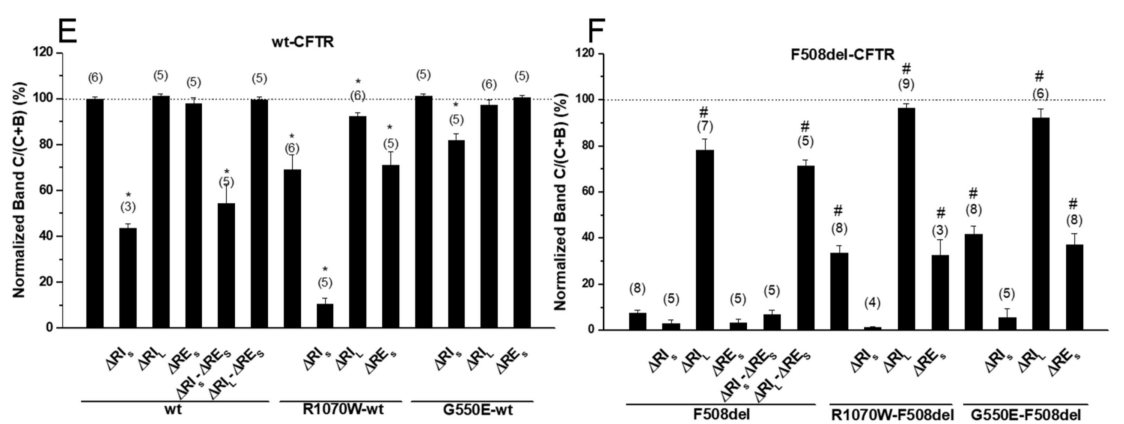

Figure 1. Effect of removal of short regulatory extension $\left(\Delta R E_{S}\right)$ on processing of wt- and F508del-Cystic Fibrosis (CF) transmembrane conductance regulator (CFTR). (A) Structural features of the CFTR nucleotide binding domain 1 (NBD1) domain. Protein Data Bank identifier (PDB ID): 2BBO. The main structural regions are color coded. F508 is represented as red spheres. G550 is represented as brown spheres. ATP is shown as sticks and $\mathrm{Mg}^{2+}$ as a pink sphere. Regulatory insertion (RI) is unstructured in the crystal structure and represented as a dotted red line. The G550E mutation in this structure was reverted in silico. (B-D) Samples from Baby Hamster Kidney (BHK) cell lines stably expressing different CFTR variants of $\triangle R_{S}$ and $\triangle R I$, the latter either in its short "S" or long " $L$ " versions (see Materials and Methods) and alone (B) or jointly with (C,D) genetic revertants, as indicated, were analyzed for CFTR protein expression by Western blot (WB) with anti-CFTR $596 \mathrm{Ab}$ and also anti-calnexin (CNX) as loading control. All samples were incubated with $3 \mu \mathrm{M}$ dimethyl sulfoxide (DMSO) for $48 \mathrm{~h}$, as controls for experiments with corrector VX-809 (see Figure 3). Summary of data of CFTR variants on the wt- (E) or F508del- (F) CFTR backgrounds, expressed as normalized ratios (band C/(band C + band B)) and as a percentage to the respective ratio for $\mathrm{wt}-\mathrm{CFTR}$ and as mean \pm standard error of the mean (SEM). $(n)$ indicates nr. of independent experiments. "** and "\#" indicate significantly different $(p<0.05)$ from wt-CFTR and F508del-CFTR, respectively. 
Table 1. Summary of Western blot quantification of original data in Figures 1-3 for different CFTR variants in the presence of VX-809 or DMSO control. Data are expressed as normalized ratios (band C/ band $(B+C)$ ) for each variant and as a percentage to wt-CFTR ratio.

\begin{tabular}{|c|c|c|c|c|c|}
\hline & & \multicolumn{2}{|c|}{ wt-Background } & \multicolumn{2}{|c|}{ F508del-Background } \\
\hline & & Control & VX-809 & Control & VX-809 \\
\hline & CFTR Variant & $\%$ to wt & $\%$ to $w t$ & $\%$ to $w t$ & $\%$ to $w \mathrm{t}$ \\
\hline & - & $100 \pm 1$ & $103 \pm 1$ & $8 \pm 1$ & $17 \pm 2$ \\
\hline \multirow{5}{*}{$\Delta \mathrm{RE}_{\mathrm{S}}$} & - & $98 \pm 2$ & $100 \pm 1$ & $3 \pm 2$ & $9 \pm 4$ \\
\hline & $\Delta \mathbf{R I}_{S}$ & $54 \pm 9$ & $93 \pm 3$ & $7 \pm 2$ & $20 \pm 4$ \\
\hline & $\Delta \mathrm{RI}_{\mathrm{L}}$ & $100 \pm 1$ & $97 \pm 2$ & $71 \pm 3$ & $96 \pm 2$ \\
\hline & R1070W & $71 \pm 6$ & $90 \pm 3$ & $33 \pm 7$ & $26 \pm 6$ \\
\hline & G550E & $101 \pm 1$ & $102 \pm 1$ & $37 \pm 5$ & $39 \pm 4$ \\
\hline \multirow{2}{*}{$\Delta \mathrm{RE}_{\mathrm{L}}$} & - & $88 \pm 5$ & $87 \pm 3$ & $9 \pm 2$ & $8 \pm 2$ \\
\hline & $\Delta \mathrm{H} 9$ & $55 \pm 3$ & $69 \pm 3$ & $0 \pm 0$ & $0 \pm 0$ \\
\hline & $\Delta \mathrm{H} 9$ & $72 \pm 4$ & $69 \pm 3$ & $1 \pm 0$ & $0 \pm 0$ \\
\hline \multirow{3}{*}{$\Delta \mathbf{R I}_{S}$} & - & $44 \pm 2$ & $90 \pm 3$ & $3 \pm 2$ & $4 \pm 3$ \\
\hline & R1070W & $11 \pm 3$ & $56 \pm 8$ & $2 \pm 0$ & $4 \pm 2$ \\
\hline & G550E & $82 \pm 3$ & $98 \pm 1$ & $6 \pm 4$ & $5 \pm 3$ \\
\hline \multirow{3}{*}{$\Delta \mathrm{RI}_{\mathrm{L}}$} & - & $101 \pm 1$ & $101 \pm 0$ & $78 \pm 5$ & $92 \pm 4$ \\
\hline & R1070W & $92 \pm 2$ & $94 \pm 3$ & $96 \pm 2$ & $96 \pm 3$ \\
\hline & G550E & $97 \pm 2$ & $92 \pm 2$ & $92 \pm 4$ & $97 \pm 2$ \\
\hline & R1070W & $69 \pm 7$ & $93 \pm 4$ & $34 \pm 3$ & $46 \pm 4$ \\
\hline & G550E & $101 \pm 1$ & $101 \pm 1$ & $42 \pm 4$ & $73 \pm 6$ \\
\hline
\end{tabular}
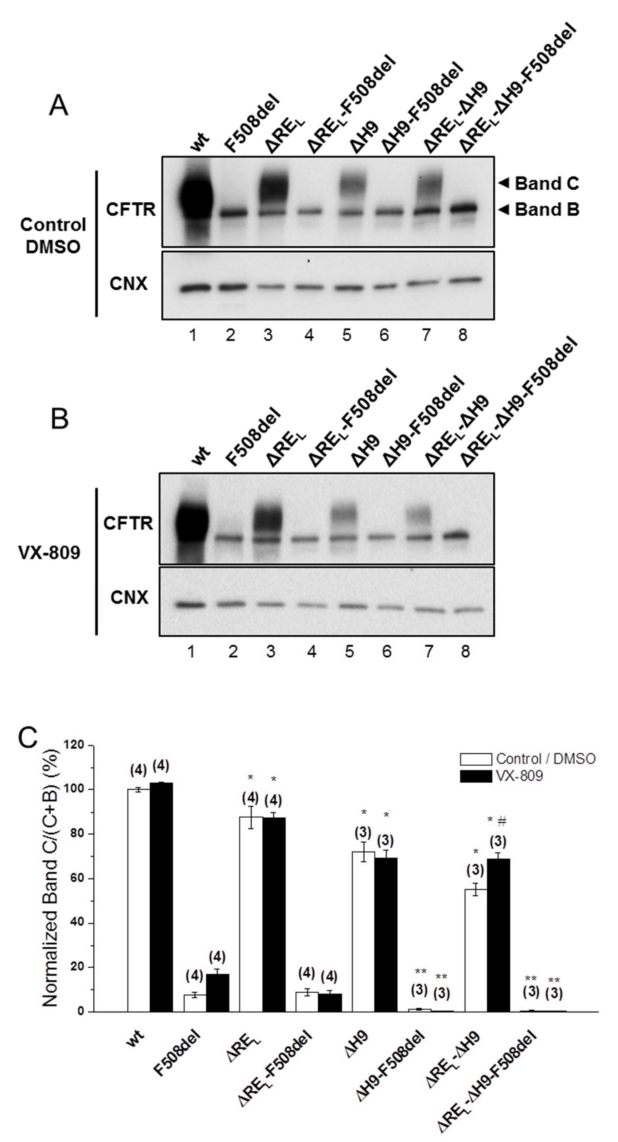

Figure 2. Effect of removal of helix $\mathrm{H} 9$ in combination with $\Delta \mathrm{RE}_{\mathrm{L}}$ and VX-809 on processing of wt- and 
F508del-CFTR. (A,B) WB analysis of samples from BHK cell lines stably expressing wt- or F508del-CFTR variants with $\Delta \mathrm{RE}_{\mathrm{L}}, \Delta \mathrm{H}$ 9, or $\Delta \mathrm{RE}_{\mathrm{L}}-\Delta \mathrm{H} 9$. Cells were incubated with $3 \mu \mathrm{M}$ VX-809 or DMSO (control) for $48 \mathrm{~h}$ at $37^{\circ} \mathrm{C}$. CFTR protein expression was analyzed by WB with the anti-CFTR 596 and anti-CNX Abs. (C) Summary of data expressed as normalized ratios (Band C / (Band C + B)) and as a percentage to the respective ratios on the wt- or F508del-CFTR backgrounds and shown as mean \pm SEM. (n) indicates number of independent experiments. "\#" indicates significantly different from the respective variant treated with DMSO. "** and "*** indicate significantly different from wt- or F508del-CFTR, respectively.
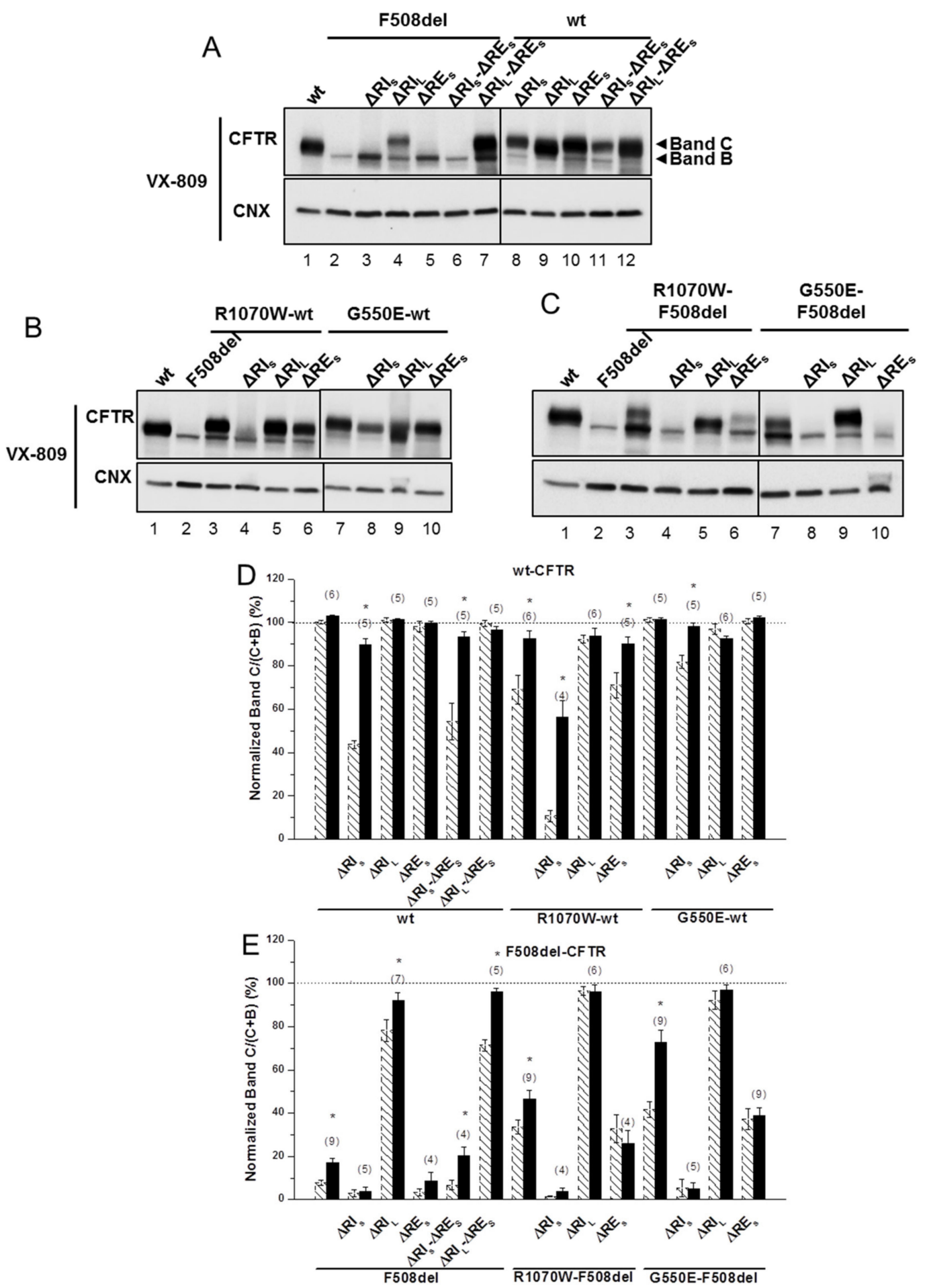

Figure 3. Effect of VX-809 on processing of wt- and F508del-CFTR variants without regulatory extension. 
(A-C) WB of samples from BHK cell lines stably expressing different CFTR variants of $\triangle \mathrm{RE}$ and $\triangle R I$, as indicated, alone (A) or jointly with $(B, C)$ genetic revertants (see Materials and Methods). CFTR protein expression was analyzed by WB with anti-CFTR 596 and anti-CNX Abs as in Figure 1. All samples were incubated with $3 \mu \mathrm{M}$ VX-809 for $48 \mathrm{~h}$. (D, E) Summary of data of variants on the wt- (D) or F508del- (E) CFTR backgrounds, expressed as normalized ratios (band C/(band C + band B)) and as a percentage to the respective ratio for wt-CFTR and as mean \pm SEM. (n) indicates number of independent experiments. "“" indicates significantly different $(p<0.05)$ from respective variant without VX-809 (as shown in Figure 1 and indicated here by dashed bars).

Removal of $\mathrm{RE}_{\mathrm{S}}$ together with $\mathrm{RI}_{\mathrm{L}}$-long RI ( $\Delta^{404} \mathrm{Gly}-\mathrm{Leu}^{435}$, Figure S1) led to an increase in processing of F508del-CFTR from $3 \pm 2 \%$ to $71 \pm 3 \%$ (vs. wt-CFTR levels), similarly to what had been previously reported by Alexandrov et al. for $\Delta \mathrm{RI}_{\mathrm{L}}$ alone [13]. In contrast, removal of $\mathrm{RI}_{\mathrm{S}}$-short $\mathrm{RI}$ $\left(\Delta^{412}\right.$ Ala-Leu ${ }^{428}$, Figure S1) had no impact on $\Delta$ RES $_{S}$-F508del-CFTR processing (Figure 1B, lanes 6,7; Figure 1F; Table 1). So in summary, removal of $\mathrm{RE}_{\mathrm{S}}$ from the $\Delta \mathrm{RI}_{\mathrm{S}}$ - or $\Delta \mathrm{RI}_{\mathrm{L}}-\mathrm{F} 508$ del-CFTR variants had no further effect on their processing, despite that processing of $\triangle \mathrm{RE}_{\mathrm{S}}-\mathrm{F} 508 \mathrm{del}-\mathrm{CFTR}$ is different upon removal of $\mathrm{RI}_{\mathrm{S}}$ or $\mathrm{RI}_{\mathrm{L}}$, but this difference remains equivalent to removal $\mathrm{RI}_{\mathrm{S}}$ or $\mathrm{RI}_{\mathrm{L}}$ alone. Despite this lack of impact on processing, removal of $\mathrm{RE}_{\mathrm{S}}$ does affect total protein expression levels of $\Delta \mathrm{RI}_{\mathrm{S}^{-}}$or $\triangle \mathrm{RI}_{\mathrm{L}}$-F508del-CFTR variants leading to a decrease in RIS-F508del-CFTR (Figure 1B, lanes 3,6) and an increase in $\mathrm{RI}_{\mathrm{L}}$-F508del-CFTR (Figure $1 \mathrm{~B}$, lanes 4,7). Interestingly, when $\mathrm{RE}_{\mathrm{S}}$ and $\mathrm{RI}_{\mathrm{S}}$, were jointly removed from wt-CFTR, its processing was significantly reduced to $54 \pm 9 \%$, while $\Delta \mathrm{RE}_{\mathrm{S}}$ jointly with $\triangle \mathrm{RI}_{\mathrm{L}}$ caused no impact (Figure 1B, lanes11,12, respectively; Figure 1E; Table 1). Again, these data were equivalent to removal of $\mathrm{RI}_{\mathrm{S}}$ or $\mathrm{RI}_{\mathrm{L}}$ alone on wt-CFTR processing (Figure 1B, lanes 8,9; Figure 1F; Table 1).

The differential effect caused by removal of $\mathrm{RI}_{\mathrm{S}}$ vs $\mathrm{RI}_{\mathrm{L}}$ on F508del- and wt-CFTR emphasize the importance of those $8 \mathrm{~N}$-term $\left({ }^{404} \mathrm{Gly}\right.$-Lys $\left.{ }^{411}\right)$ and $7 \mathrm{C}$-term $\left(\mathrm{Phe}^{429}\right.$-Leu $\left.{ }^{435}\right)$ amino acid residues that differ between the two RI regions for the folding and processing of CFTR.

Overall, removal of $\mathrm{RE}_{\mathrm{S}}$ alone or with RI has no impact on F508del-CFTR processing efficiency.

2.2. Simultaneous Removal of Long Regulatory Extension $\left(R E_{L}\right)$ and Helix H9 Significantly Reduces wt-CFTR Processing but Increases Levels of F508del-CFTR Immature Form

Next, we assessed the impact of removing the long version of RE-RE ${ }_{L}\left(\Delta^{647} C y s-S^{6}{ }^{678}\right.$, Figure 2)which significantly decreased wt-CFTR processing to $88 \pm 5 \%$ (Figure 2A, lane 3; Figure 2C) but had no impact on F508del-CFTR (Figure 2A, lane 4; Figure 2C). As helix H9 $\left(\Delta^{637} \mathrm{Gln}-\mathrm{Gly}{ }^{646}\right.$, Figure S1) can be considered to be part of this longer RE region [18] since it interacts with RE (Figure 1A), we also tested the effect of removing helix $\mathrm{H} 9$ jointly with $\triangle R E_{\mathrm{L}}$. Deletion of both $\mathrm{H} 9(\Delta \mathrm{H} 9)$ and $R E_{\mathrm{L}}$ further decreased the residual processing of $\Delta \mathrm{RE}_{\mathrm{L}}$-F508del-CFTR from $9 \pm 2 \%$ to $0 \pm 0 \%$ and in fact the same happened for $\Delta \mathrm{H} 9$ alone (Figure 2A, lanes 8,6, respectively). Curiously, however, levels of immature F508del-CFTR were significantly increased when both $\mathrm{RE}_{\mathrm{L}}$ and $\mathrm{H} 9$ were removed (Figure 2A, lane 8), similarly to $\triangle \mathrm{RE}_{\mathrm{S}}$-F508del-CFTR (Figure $1 \mathrm{~B}$ ) and in contrast to $\triangle \mathrm{RE}_{\mathrm{L}}-\mathrm{F} 508 \mathrm{del}-\mathrm{CFTR}$.

For wt-CFTR, $\Delta \mathrm{RE}_{\mathrm{L}}-\Delta \mathrm{H} 9$ also led to a decrease in processing (55 $\pm 3 \%$ ), which was more pronounced than for $\triangle \mathrm{RE}_{\mathrm{L}}$ alone, but interestingly removal of $\mathrm{H} 9$ helix alone only reduced processing to $72 \pm 4 \%$ (Figure $2 \mathrm{~A}$, lanes 7,5 , respectively).

Simultaneous removal of $\mathrm{RE}_{\mathrm{L}}$ and helix $\mathrm{H} 9$ significantly reduces wt-CFTR processing but increases levels of F508del-CFTR immature form.

\section{3. $\Delta R I_{S}$, but Not $\Delta R E_{S}$, Abolishes the Plasma Membrane Rescue of F508del-CFTR by Revertants}

In order to test whether the stabilized immature form of $\triangle \mathrm{RE}_{\mathrm{S}}$-F508del-CFTR could be rescued to mature form, we next tested the effects of removing the $\mathrm{RE}_{S}$ from F508del-CFTR with R1070W and G550E revertants. Indeed, both the R1070W and G550E revertants rescued $\triangle \mathrm{RE}_{\mathrm{S}}$-F508del-CFTR to $33 \pm 7 \%$ and $37 \pm 5 \%$, respectively (Figure 1D, lanes 6,10; Table 1 ). 
However, removal of $\mathrm{RI}_{\mathrm{S}}$ from F508del-CFTR with R1070W and G550E revertants, virtually abolished the rescue of F508del-CFTR by both revertants (Figure 1D, lanes 4,8; Table 1). Moreover, processing of $\Delta \mathrm{RI}_{\mathrm{L}}$-F508del-CFTR was further increased by either $\mathrm{R} 1070 \mathrm{~W}$ or G550E up to $96 \pm 2 \%$ and $92 \pm 4 \%$, respectively (Figure 1D, lanes 5,9; Table 1). Alone, R1070W and G550E rescued F508del-CFTR from $8 \pm 1 \%$ to $34 \pm 3 \%$ and $42 \pm 4 \%$, respectively (Figure 1D, lanes 3,7; Table 1), as we previously reported [5].

Interestingly, the impaired processing of $\Delta \mathrm{RI}_{\mathrm{S}}$-wt-CFTR (44 $\left.\pm 2 \%\right)$ was significantly rescued by G550E (to $82 \pm 3 \%$ ), but curiously it was further reduced by R1070W (to $11 \%$ ) i.e., close to levels of F508del-CFTR processing (Figure 1C, lanes 8,4; Table 1). Of note that R1070W alone also reduced wt-CFTR processing to $69 \pm 7 \%$, but this reduction could be compensated by the removal of $\mathrm{RI}_{\mathrm{L}}$ (92 $\pm 2 \%$ ) (Figure 1C, lanes 3,5; Table 1), while G550E alone caused no effect on wt-CFTR processing (Figure 1C, lane 7).

In summary, $\Delta \mathrm{RI}_{\mathrm{S}}$, but not $\Delta \mathrm{RE}_{\mathrm{S}}$, abolishes the plasma membrane rescue of F508del-CFTR by genetic revertants R1070W and G550E.

\section{4. $\Delta R I_{L}$ Synergises with VX-809, but Not with Revertants to Rescue $\Delta R E_{S}$-F508del-CFTR Processing}

To further test how the stabilized immature form of $\Delta \mathrm{RE}_{\mathrm{S}}$-F508del-CFTR could be pharmacologically rescued, we then assessed the impact of corrector VX-809 (which per se promotes maturation of the F508del-CFTR) on the processing of this and of the other variants, to obtain structural insight on the effects of this novel drug. Although $\triangle \mathrm{RE}_{\mathrm{S}}$-F508del-CFTR could not be rescued by VX-809, data show that this small molecule was able to rescue $\Delta \mathrm{RI}_{\mathrm{S}}-\Delta \mathrm{RE}_{\mathrm{S}}$-F508del-CFTR (from $7 \pm 2 \%$ to $20 \pm 4 \%$ ) and further increased processing of $\Delta \mathrm{RI}_{\mathrm{L}}-\Delta \mathrm{RE}_{\mathrm{S}}$-F508del-CFTR to wt-CFTR levels (from $71 \pm 3 \%$ to $96 \pm 2 \%$ ) (Figure 3A, lanes 6,7; Figure 3E; Table 1). These data suggest a strong synergistic effect between VX-809 and $\Delta \mathrm{RI}_{\mathrm{L}}$ (and with $\Delta \mathrm{RI}_{\mathrm{S}}$, albeit to a lesser extent) to rescue $\triangle \mathrm{RE}_{\mathrm{S}}-\mathrm{F} 508 \mathrm{del}-\mathrm{CFTR}$ processing. This is particularly interesting because VX-809 did not rescue processing of the $\Delta \mathrm{RE}_{\mathrm{S}^{-}}$, nor $\Delta \mathrm{RI}_{\mathrm{S}^{-}}-\mathrm{F} 508 \mathrm{del}-\mathrm{CFTR}$ variants (Figure 3A, lanes 5,3; Table 1 ) while it recovered the processing of $\Delta \mathrm{RI}_{\mathrm{L}}$-F508del-CFTR to wt-CFTR levels (Figure 3A, lane4; Table 1). Notably, VX-809 was able to almost completely revert processing impairment of $\Delta \mathrm{RI}_{\mathrm{S}}$-wt-CFTR and $\Delta \mathrm{RI}_{\mathrm{S}}-\Delta \mathrm{RE}_{\mathrm{S}}$-CFTR (from $44 \pm 2 \%$ and $54 \pm 9 \%$ ) to $90 \pm 3 \%$ and $93 \pm 3 \%$, respectively (Figure $3 \mathrm{~A}$, lanes 8,11 ; Figure 3D; Table 1).

Strikingly, VX-809 had no effect on processing of $\triangle \mathrm{RE}_{\mathrm{S}}$-F508del-CFTR with R1070W or G550E (Figure 3C, lanes 6,10; Figure 3E; Table 1), while it further rescued processing of F508del-CFTR variants with R1070W or G550E alone to $46 \pm 4 \%$ and $73 \pm 6 \%$, respectively (Figure 3C, lanes 3,7; Figure 3E; Table 1), as reported [5]. Similarly, VX-809 caused no significant further rescue on the processing of $\triangle \mathrm{RI}_{\mathrm{L}}$-F508del-CFTR with those revertants, but these variants already had processing levels close to those of wt-CFTR (Figure 3C, lanes 5,9; Figure 3E; Table 1). Interestingly, the impaired processing of $\triangle \mathrm{RE}_{\mathrm{S}}-\mathrm{R} 1070 \mathrm{~W}$-wt-CFTR $(71 \pm 6 \%)$ was also reverted by VX-809 to $90 \pm 3 \%$ (Figure 3B, lane 6; Table 1).

Corrector VX-809 failed to rescue any of the $\Delta \mathrm{RE}_{\mathrm{L}^{-}}, \Delta \mathrm{H} 9-$, and $\Delta \mathrm{RE}_{\mathrm{L}}-\Delta \mathrm{H} 9-\mathrm{F} 508 \mathrm{del}-\mathrm{CFTR}$ variants (Figure $2 \mathrm{~B}, \mathrm{C}$ ), while significantly increasing the processing of the $\Delta \mathrm{RE}_{\mathrm{L}}-\Delta \mathrm{H} 9$-wt-CFTR from $55 \pm 3 \%$ to $69 \pm 3 \%$ (Figure 2B, lane 7; Figure 2C).

Altogether, these results suggest that $\mathrm{RI}_{\mathrm{L}}$ and the genetic revertants interfere with the rescue of F508del-CFTR by VX-809.

\section{5. $\Delta R I_{L}-\Delta R E_{S}-F 508 d e l-C F T R$ Levels at the Plasma Membrane are Equivalent to Those of wt-CFTR}

To determine the fraction of the above CFTR variants that localize to the PM, we used quantitative cell surface biotinylation. These data showed that PM levels of $\Delta \mathrm{RI}_{\mathrm{L}}-\Delta R E_{\mathrm{S}}-\mathrm{F} 508 \mathrm{del}-\mathrm{CFTR}$ were equivalent to those of wt-CFTR, while those of $\Delta \mathrm{RI}_{\mathrm{L}}$-F508del-CFTR were significantly lower (Figure $4 \mathrm{~A}$, lanes 5,4; Figure $4 \mathrm{~B}$ ). Data also confirmed that $\triangle R E_{S}$ did not induce appearance of F508del-CFTR at the cell surface (data not shown). Corrector VX-809 further increased the PM expression of $\Delta \mathrm{RI}_{\mathrm{L}}-\Delta \mathrm{RE}_{\mathrm{S}}-\mathrm{F} 508 \mathrm{del}-\mathrm{CFTR}$ to levels that are significantly higher than those of wt-CFTR (Figure $4 \mathrm{~A}$, 
lanes 2,9, Figure $4 \mathrm{~B}$ ). This compound also significantly increased PM levels of $\Delta \mathrm{RI}_{\mathrm{L}}$-F508del-CFTR to similar levels of wt-CFTR (Figure 4A, lanes 2,8; Figure 4B).

A

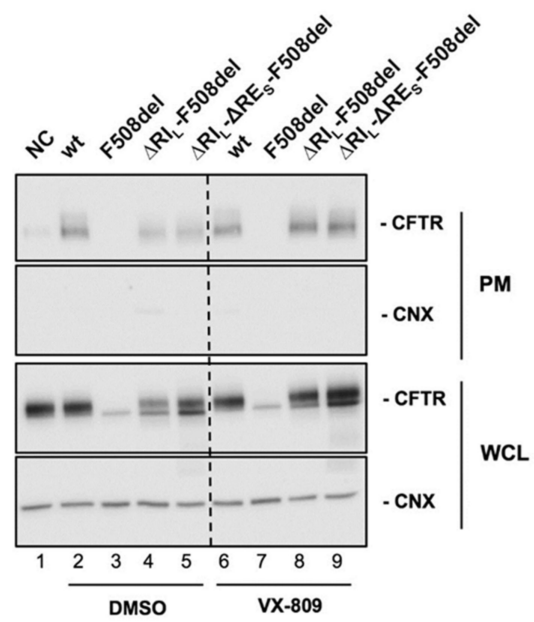

B

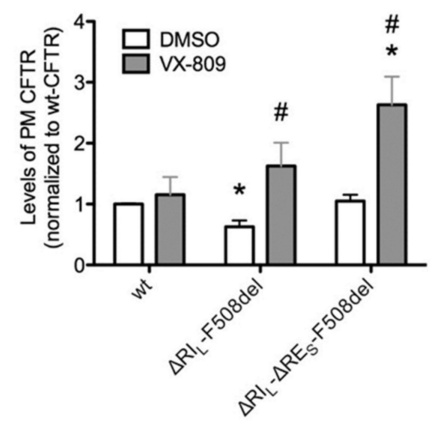

C
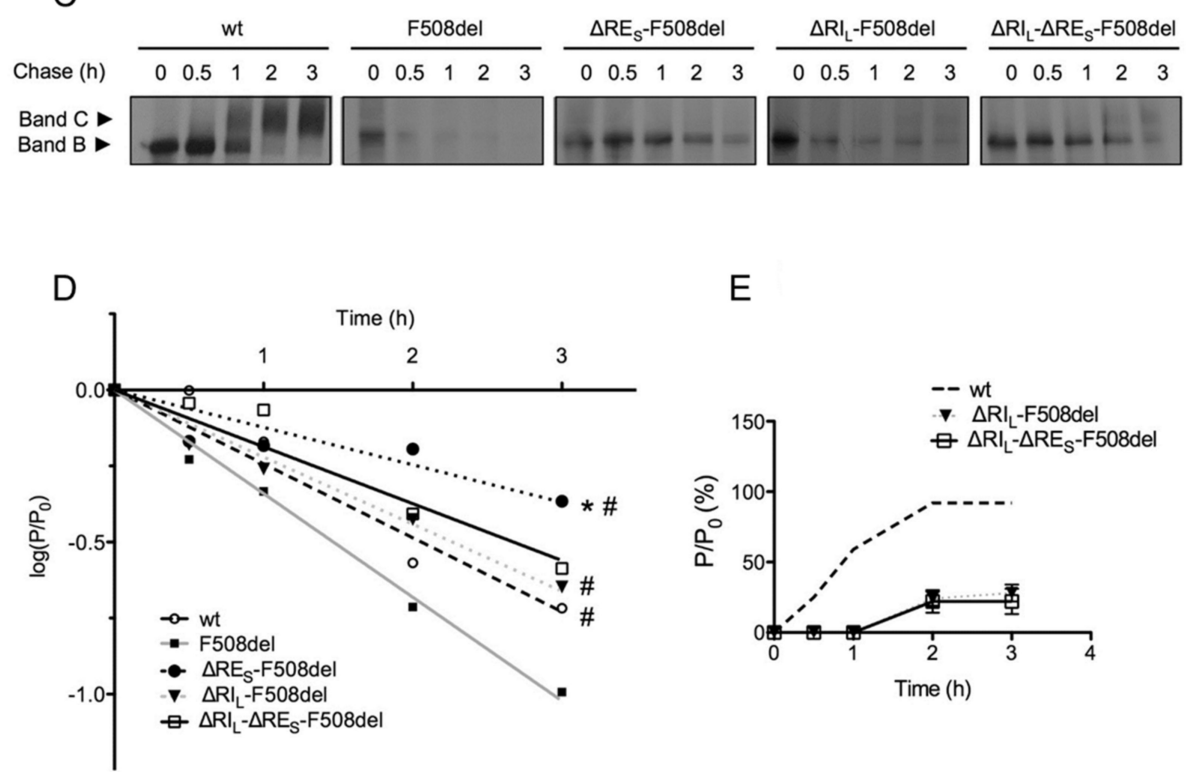

E

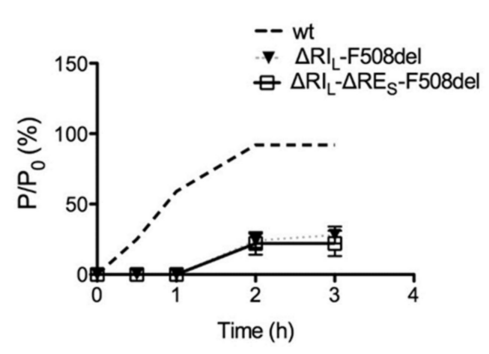

Figure 4. Plasma membrane levels, efficiency of processing and turnover of immature CFTR without Regulatory Extension $\left(R E_{S}\right)$. (A) BHK cells expressing $\Delta \mathrm{RI}_{\mathrm{L}}-\mathrm{F} 508 \mathrm{del}$ and $\Delta \mathrm{RI}_{\mathrm{L}}-\Delta \mathrm{RE}_{\mathrm{S}}-\mathrm{F} 508 \mathrm{del}-\mathrm{CFTR}$ and treated with $3 \mu \mathrm{M}$ VX-809 for $48 \mathrm{~h}$ (or DMSO control) were subjected to cell surface biotinylation. wt-CFTR samples not treated with biotin were the negative control (NC). After streptavidin pull-down, CFTR was detected by WB. CFTR and CNX are detected in the whole cell lysate (WCL) as controls. (B) Quantification of data in (a) for PM CFTR normalized to total protein and shown as fold change relatively to wt-CFTR cells treated with DMSO. "**" and "\#" indicate significantly different from wt-CFTR treated with DMSO and from respective variant without VX-809, respectively $(p<0.05)$. (C) BHK cells expressing wt-, F508del-CFTR alone or jointly with $\Delta \mathrm{RI}_{\mathrm{L}}, \Delta \mathrm{RE}_{\mathrm{S}}$, and $\Delta \mathrm{RI}_{\mathrm{L}}-\Delta \mathrm{RE}_{\mathrm{S}}$ were subjected to pulse-chase (see Materials and Methods) for the indicated times (0, 0.5, 1, 2, and $3 \mathrm{~h}$ ) before lysis and immunoprecipitation (IP) with the anti-CFTR $596 \mathrm{Ab}$. After electrophoresis and fluorography, images were analyzed by densitometry. (D) Turnover of immature (band B) CFTR for different CFTR variants is shown as the percentage of immature protein at a given time point of chase $(\mathrm{P})$ relative to the amount at $t=0\left(\mathrm{P}_{0}\right)$. (E) Efficiency of processing of band $B$ into band $C$ is shown as the percentage of band $C$ at a given time of chase relative to the amount of band $\mathrm{B}$ at $\mathrm{t}=0$. " "** and "\#" indicate statistical significantly different $(p<0.05)$ from wt-CFTR and F508del-CFTR, respectively. Data represent mean $\pm \operatorname{SEM}(n=5)$. 
Given the very significant stabilization of immature $\Delta \mathrm{RE}_{\mathrm{S}}$-F508del-CFTR (Figure 1B), next, we determined how removal of $R_{S}$ affected the processing efficiency and the turnover of the F508deland $\Delta \mathrm{RI}_{\mathrm{L}}$-F508del-CFTR variants. To this end, we performed pulse-chase experiments (Figure $4 \mathrm{C}, \mathrm{D}$ ) and indeed our results revealed that $\triangle \mathrm{RE}_{\mathrm{S}}$ very significantly stabilized immature F508del-CFTR not just relatively to F508del-CFTR but to levels even significantly higher than those of wt-CFTR (Figure 4C,D). Indeed, our data show that although F508del-CFTR without RE did not traffic to the PM, it showed a dramatic stabilization of its immature form (evidencing a turnover rate $\sim 2 \times$ lower than that of wt-CFTR. It should be noted that the turnover of F508del-CFTR itself is $\sim 1.4 \times$ faster vs. wt-CFTR, thus this stabilization (of $\sim 3 \times$ vs. F508del-CFTR) represents indeed a massive stabilization. Interestingly, this stabilizing effect was no longer significant for $\Delta \mathrm{RI}_{\mathrm{L}}-\Delta R E_{\mathrm{S}}-\mathrm{F} 508 \mathrm{del}-\mathrm{CFTR}$ nor for $\Delta \mathrm{RI}_{\mathrm{L}}-\mathrm{F} 508$ del-CFTR, the latter being equivalent to wt-CFTR (Figure $4 \mathrm{C}, \mathrm{D}$ ). Removal of $\mathrm{RI}_{\mathrm{S}}$ from F508del-CFTR did not stabilize its turnover (data not shown). As to removal of either $\mathrm{RE}_{\mathrm{S}}$ or $\mathrm{RI}_{\mathrm{S}}$ from wt-CFTR, it did not affect the processing efficiency or the turnover vs. wt-CFTR (Figure S2).

In summary, levels of $\Delta \mathrm{RI}_{\mathrm{L}}-\Delta \mathrm{RE}_{\mathrm{S}}-\mathrm{F} 508 \mathrm{del}$-CFTR at the PM are equivalent to those of wt-CFTR.

\subsection{VX-809 Jointly with RE and RI Removal Completely Restored F508del-CFTR Function as Chloride Channel}

To investigate the channel function of the $\Delta \mathrm{RE}_{\mathrm{S}}$ - and $\Delta \mathrm{RI}_{\mathrm{L}}$-F508del-CFTR variants, we used the iodide efflux technique. Removal of $\mathrm{RE}_{\mathrm{S}}$ alone had no impact on F508del-CFTR function (Figure 5A; black dash line in Figure S3B) as expected from the lack of processed form for this variant (Figure 1B). However, when $\mathrm{RE}_{\mathrm{S}}$ and $\mathrm{RI}_{\mathrm{L}}$ were removed jointly from F508del-CFTR, functional levels reached $78 \pm 7 \%$ of wt-CFTR (Figure 5A,C; black dotted line in Figure S3B). As to removal of $\mathrm{RI}_{\mathrm{L}}$ alone from F508del-CFTR, it restored function to $92 \pm 7 \%$ of wt-CFTR (Figure 5A,C; black line in Figure S3B), as described [13]. Also, the delay in peak response observed for F508del-CFTR ( $\mathrm{min}=4)$ vs. wt-CFTR $(\min =2)$, was partially corrected (to $\min =3$ ) both by removal of $\mathrm{RI}_{\mathrm{L}}$ alone from F508del-CFTR or together with $\mathrm{RE}_{\mathrm{S}}$ (Figure 5C; Figure S3B, black line and dotted line).

Introduction of G550E and R1070W revertants into the $\Delta \mathrm{RI}_{\mathrm{L}}$-F508del-CFTR variant slightly but not significantly decreased its function from $92 \pm 7 \%$ to $71 \pm 8 \%$ and $76 \pm 8 \%$, respectively (Figure $5 \mathrm{~A}, \mathrm{C}$; black lines in Figure S3C,D), in parallel to the respective processing levels (Figure 1). In fact, these variants showed higher activity levels than the revertants containing $\mathrm{RI}_{\mathrm{L}}(29 \pm 3 \%$ and $56 \pm 6 \%$, respectively). Interestingly however, both G550E- and R1070W- $\Delta \mathrm{RI}_{\mathrm{L}}-\mathrm{F} 508 \mathrm{del}-\mathrm{CFTR}$ fully corrected (to min $=2$ ) the delay in peak response of F508del-CFTR, which was still partially present in $\Delta \mathrm{RI}_{\mathrm{L}^{-}}$ and $\Delta \mathrm{RI}_{\mathrm{L}}-\mathrm{RE}_{\mathrm{S}}$-F508del-CFTR $(\mathrm{min}=3)$.

The effect of VX-809 $\left(3 \mu \mathrm{M}, 48 \mathrm{~h}\right.$ at $\left.37{ }^{\circ} \mathrm{C}\right)$ was also examined at functional level on the $\Delta \mathrm{RE}_{\mathrm{S}^{-}}, \Delta \mathrm{RI}_{\mathrm{L}^{-}}-\mathrm{RE}_{\mathrm{S}^{-}}$and $\Delta \mathrm{RI}_{\mathrm{L}}-\mathrm{F} 508$ del-CFTR variants. VX-809 shows a tendency to increase in $\Delta \mathrm{RE}_{\mathrm{S}}-\Delta \mathrm{RI}_{\mathrm{L}}$-F508del-CFTR function ( $84 \pm 8 \%$ of wt-CFTR) which was parallel to the observed increase in PM expression (Figure 4; Figure 5A,C; black dotted line in Figure S3F). Also, $\Delta \mathrm{RE}_{\mathrm{S}}-\Delta \mathrm{RI}_{\mathrm{L}}-$ F508del-CFTR fully corrected (to $\min =2$ ) the delay in peak response of F508del-CFTR which was still partially present in $\Delta \mathrm{RI}_{\mathrm{L}}$ - and $\Delta \mathrm{RI}_{\mathrm{L}}-\Delta \mathrm{RE}_{\mathrm{S}}-\mathrm{F} 508 \mathrm{del}-\mathrm{CFTR}(\mathrm{min}=3)$.

In contrast to the observed increase in processing, VX-809 caused no significant increase in the function of $\triangle \mathrm{RI}_{\mathrm{L}}$-F508del-CFTR and actually a slight, but not significant decrease was observed and there was still the same delay in peak response (Figure 5A,C; black line in Figure S3F).

A similar slight decrease in function was observed for VX-809 on the $\Delta \mathrm{RI}_{\mathrm{L}}-\mathrm{R} 1070 \mathrm{~W}-\mathrm{F} 508 \mathrm{del}-\mathrm{CFTR}$ variant (Figure 5A,C; black line in Figure $\mathrm{S3H}$ ), but the most striking result was the significant decrease caused by VX-809 on function of $\Delta \mathrm{RI}_{\mathrm{L}}$-G550E-F508del from $76 \pm 8 \%$ to $41 \pm 3 \%$ (Figure $5 \mathrm{~A}$; black line in Figure S3G). Nevertheless, both revertant variants of $\Delta \mathrm{RI}_{\mathrm{L}}$-F508del-CFTR showed no change in peak response which was still the same as wt-CFTR $(\min =2)$.

Overall, VX-809 jointly with RE and RI removal completely restored F508del-CFTR function as chloride channel. 

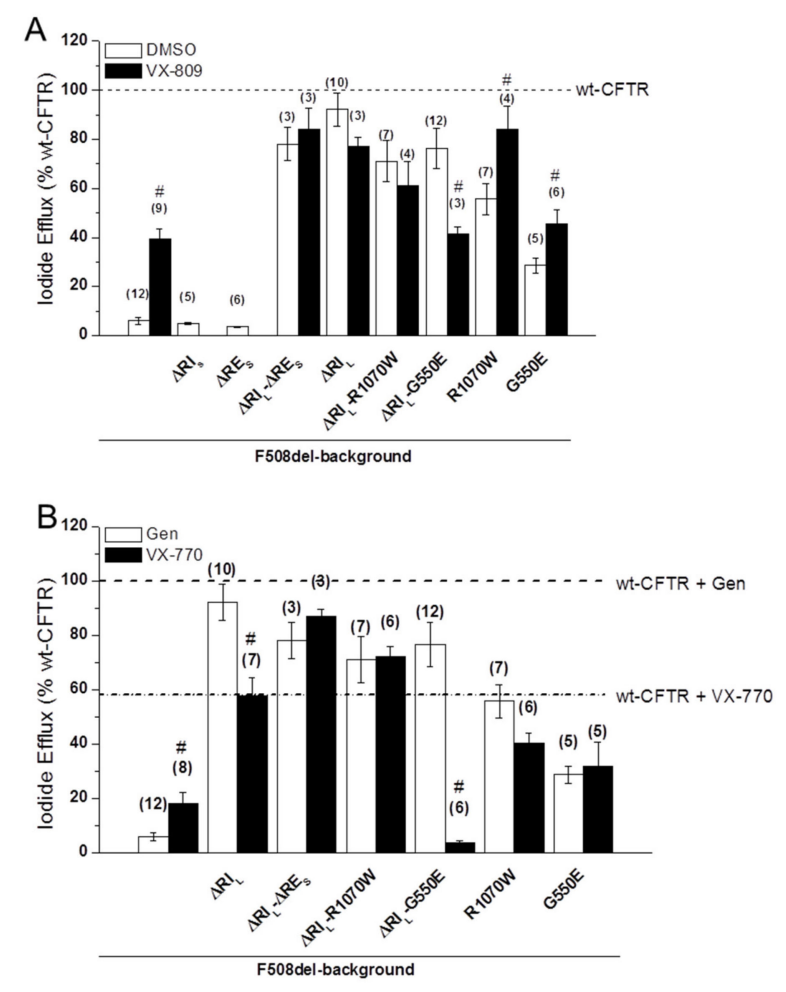

\begin{tabular}{|c|c|c|c|c|c|c|c|c|c|}
\hline \multirow[b]{2}{*}{ CFTR variant } & \multicolumn{3}{|c|}{ DMSO } & \multicolumn{3}{|c|}{ VX-809 } & \multicolumn{3}{|c|}{ VX-770 } \\
\hline & peak $(\min )$ & value & $\begin{array}{c}\text { \% to control } \\
\text { wt-CFTR }\end{array}$ & peak (min) & ) value & $\begin{array}{c}\text { \% to control } \\
\text { wt-CF TR }\end{array}$ & peak (min) & value & $\begin{array}{c}\text { \% to control } \\
\text { wt-CFTR }\end{array}$ \\
\hline wt & 2 & $51 \pm 3$ & $100 \pm 5$ & 2 & $51 \pm 4$ & $100 \pm 8$ & 2 & $30 \pm 4$ & $58 \pm 9$ \\
\hline F508del & 4 & $3 \pm 1$ & $6 \pm 1$ & 4 & $20 \pm 2$ & $39 \pm 4$ & 4 & $9 \pm 2$ & $18 \pm 4$ \\
\hline$\Delta \mathrm{RI}_{\mathrm{S}} \mathrm{F}$ 508del & . & $2 \pm 0$ & $5 \pm 0$ & . & . & - & . & - & - \\
\hline$\Delta \mathrm{RE}_{\mathrm{S}}-\mathrm{F} 508 \mathrm{del}$ & . & $2 \pm 0$ & $3 \pm 0$ & . & . & . & . & . & . \\
\hline$\Delta R I_{L}-\Delta R E_{S}-F 508 \mathrm{del}$ & 3 & $40 \pm 3$ & $78 \pm 7$ & 2 & $43 \pm 4$ & $84 \pm 8$ & 2 & $44 \pm 1$ & $87 \pm 3$ \\
\hline$\Delta \mathrm{RI}_{\mathrm{L}}$-F508del & 3 & $47 \pm 3$ & $92 \pm 7$ & 3 & $39 \pm 2$ & $77 \pm 4$ & 3 & $30 \pm 3$ & $58 \pm 7$ \\
\hline$\Delta R I_{L}-R 1070$ W-F508del & 2 & $36 \pm 4$ & $71 \pm 8$ & 2 & $31 \pm 5$ & $61 \pm 10$ & 1 & $37 \pm 2$ & $72 \pm 4$ \\
\hline${ }_{\Delta} \mathrm{RI}_{\mathrm{L}}$-G550E-F508del & 2 & $39 \pm 4$ & $76 \pm 8$ & 2 & $21 \pm 2$ & $41 \pm 3$ & . & $2 \pm 0$ & $4 \pm 1$ \\
\hline R1070W-F508del & 2 & $28 \pm 3$ & $56 \pm 6$ & 2 & $43 \pm 5$ & $84 \pm 10$ & 3 & $21 \pm 2$ & $40 \pm 4$ \\
\hline G550E-F508deI & 2 & $15 \pm 2$ & $29 \pm 3$ & 2 & $23 \pm 3$ & $46 \pm 5$ & 3 & $16 \pm 4$ & $32 \pm 9$ \\
\hline
\end{tabular}

Figure 5. Functional characterization of the $\Delta \mathrm{RE}_{\mathrm{s}}$ and $\Delta \mathrm{RI}_{\mathrm{l}}$ variants of F508del-CFTR with or without VX-809 or VX-770 treatments. (A,B) Summary of the data from the iodide efflux peak magnitude generated by BHK cells stably expressing different CFTR variants and treated with $3 \mu \mathrm{M}$ VX-809 (black bars on panel A) or with DMSO as control (white bars on panel a) for $48 \mathrm{~h}$ and stimulated with $10 \mu \mathrm{M}$ Forskolin (Fsk) and $50 \mu \mathrm{M}$ Genistein (Gen). In (B) cells were not pre-incubated and were stimulated either with $10 \mu \mathrm{M}$ Fsk and $50 \mu \mathrm{M}$ Gen (white bars on panel B) or with $10 \mu \mathrm{M}$ Fsk and $10 \mu \mathrm{M}$ VX-770 (black bars on panel b), as indicated. Data are shown as a percentage of wt-CFTR activity under Fsk/Gen stimulation and as mean \pm SEM. (n) indicates number of independent experiments. "\#" indicates significantly different $(p<0.05)$ from the same CFTR variant incubated with DMSO (A) or significantly different from the same CFTR variant under Fsk/Gen stimulation (white bars) (B). (C) Summary of iodide efflux data for different F508del-CFTR variants without $\mathrm{RE}_{\mathrm{S}}, \mathrm{RI}_{\mathrm{s}}$, and $\mathrm{RI}_{\mathrm{l}}$ alone or jointly with revertants R1070W and G550E.

\subsection{VX-770-Stimulated Currents of CFTR Variants are Dramatically Decreased by $\Delta R I_{L}$ but not by $\triangle R I_{L^{-}}-\Delta R E_{S}$}

Given the interesting and diverse results observed for the $\Delta \mathrm{RE}_{\mathrm{S}}$ and $\Delta \mathrm{RI}_{\mathrm{L}}$ under VX-809, next we tested the effects of potentiator VX-770 (Ivacaftor), an approved drug for CF patients with gating mutations (Figure 5B) on these variants and in combination with VX-809, for F508del/F508del patients. Most strikingly, our data show that VX-770/Forskolin (Fsk) significantly decreased the function of $\Delta \mathrm{RI}_{\mathrm{L}}$-F508del-CFTRin comparison with potentiation by Gen/Fsk (Figure $5 \mathrm{~B}, \mathrm{C}$ ) from $92 \pm 7 \%$ to $58 \pm 7 \%$ of wt-CFTR (Figure 5B,C; black line in Figure S3J). As $\triangle$ RE -F508del-CFTR was not processed, we did 
not test the effect of VX-770 on this variant, but when the $\Delta \mathrm{RE}_{\mathrm{S}}-\Delta \mathrm{RI}_{\mathrm{L}}-\mathrm{F} 508$ del-CFTR variant was assessed for its function after acute application of VX-770 with Fsk, a tendency for increase in its function (to $87 \pm 3 \%$ of wt-CFTR) was observed versus its function under Gen/Fsk (78 $\pm 7 \%$ of wt-CFTR) (Figure 5B,C; black dotted line in Figure S3J).

The most dramatic result, however, was observed for the $\Delta \mathrm{RI}_{\mathrm{L}}$-G550E-F508del-CFTR variant which under VX-770/Fsk only had $4 \pm 1 \%$ function of wt-CFTR, while under Gen/Fsk it had $76 \pm 8 \%$ (Figure 5B,C; black line in Figure S3K). For $\triangle \mathrm{RI}_{\mathrm{L}}-\mathrm{R} 1070 \mathrm{~W}-\mathrm{F} 508$ del-CFTR this decrease was not observed (Figure 5B,C; black line in Figure S3L), and in fact this variant had a slightly higher function under VX-770 stimulation $\left(72 \pm 4 \%\right.$ ) than $\Delta \mathrm{RI}_{\mathrm{L}}$-F508del-CFTR (58 $\pm 7 \%$ ) (Figure $5 \mathrm{~B}, \mathrm{C}$, black line in Figure $\mathrm{S} 3 \mathrm{H}$ ). These results suggest that somehow removal of $\mathrm{RI}_{\mathrm{L}}$ can increase the chances of VX-770 blocking the channel pore.

The delay in peak response of F508del- comparing with wt-CFTR was not corrected for $\Delta \mathrm{RI}_{\mathrm{L}}$-F508del-CFTR $(\mathrm{min}=3)$, but it was corrected for $\Delta \mathrm{RI}_{\mathrm{L}}-\Delta \mathrm{RE}_{\mathrm{S}}-\mathrm{F} 508 \mathrm{del}-\mathrm{CFTR}(\min =2)$ (black and dotted black lines in Figure S3J, respectively). Interestingly, on the other hand $\Delta \mathrm{RI}_{\mathrm{L}}-\mathrm{R} 1070 \mathrm{~W}-\mathrm{F} 508 \mathrm{del}-\mathrm{CFTR}$ showed the quickest peak response (at $\mathrm{min}=1$ ), in contrast to either R1070W-F508del-CFTR or $\triangle \mathrm{RI}_{\mathrm{L}}$-F508del-CFTR, both at $\mathrm{min}=3$.

Similarly, VX-770 also significantly decreased the function of $\Delta \mathrm{RI}_{\mathrm{L}}-\mathrm{wt}-\mathrm{CFTR}$ from $127 \pm 15 \%$ under Gen to $57 \pm 6 \%$ (Figure 54 ).

These results imply that, VX-770-stimulated currents of CFTR variants are dramatically decreased by $\Delta \mathrm{RI}_{\mathrm{L}}$ but not by $\Delta \mathrm{RI}_{\mathrm{L}}-\Delta \mathrm{RE}_{\mathrm{S}}$.

\section{Discussion}

The main goal of this study was to understand how the removal of the regulatory extension $(\triangle \mathrm{RE})$ alone or with the regulatory insertion $(\Delta \mathrm{RI})$ - two highly conformationally dynamic regions-impact the rescue of F508del-CFTR processing and function by two compounds-VX-809 and VX-770 - which in combination were recently approved to clinically treat F508del-homozygous patients. Indeed, CFTR is the sole ABC transporter that functions as a channel and thus, these highly dynamic RI and $\mathrm{RE}$ regions which are absent in other $\mathrm{ABC}$ transporters, may be of high relevance to understand how CFTR differs from other ABCs, namely in its function as a channel.

These regions RI and RE were originally suggested to be positioned to impede formation of the NBD1-NBD2 dimer required for channel gating $[15,19]$. Indeed, both RI and RE were shown to be mobile elements in solution that bind transiently to the core of NBD in the $\beta$-sheet and $\alpha / \beta$ subdomains of NBD1 [3]. Similarly to what demonstrated for RI [13], it is plausible to posit that the dynamic flexibility of the RE may also result in exposure of hydrophobic surfaces thus contributing to the dynamic instability of NBD1 and thus contribute to the low folding efficiency of F508del-CFTR. The RE is a 30-residue segment at NBD1 C-terminus, so-called because it goes beyond canonical ABC NBDs [15]. Although this region was absent from the solved CFTR-NBD1 crystal structure [19], it was described as a helix packing against NBD1 at the NBD1:NBD2 interface and NMR data showed it has significant conformational flexibility $[3,21]$. Notwithstanding, there is some controversy regarding the RE boundaries. The RE (previously called H9c) was defined as ${ }^{655} \mathrm{Ala}^{-S_{e r}}{ }^{670}[15,19],{ }^{639} \mathrm{Asp}^{6} \mathrm{Ser}^{670} 20$, or ${ }^{654}$ Ser-Gly ${ }^{673}$ [3]. According to the crystal structure (PDB ID 2PZE), NBD1 extends only to ${ }^{646} \mathrm{Gly}$, where RD begins, so RE could be proposed to start at ${ }^{647} \mathrm{Cys}[3,18]$, thus being considered as part of RD, the latter absent in other $\mathrm{ABC}$ transporters [1]. As for the RI (previously called S1-S2 loop) its limits are also variable, being firstly described as a $\sim 35$-residue segment $\left({ }^{405} \mathrm{Phe}-{ }^{436} \mathrm{Leu}\right)$ consisting of $\alpha$-helices $\mathrm{H} 1 \mathrm{~b}$ and H1c [15] (Figure S1). Later, however, these limits were proposed to be ${ }^{404} \mathrm{Gly}^{-L_{e u}}{ }^{435}[13,20]$ or ${ }^{405}$ Phe-Leu ${ }^{436}[18]$.

\subsection{Impact of RE and RI on CFTR Processing and Function}

Our data shown here on CFTR variants depleted of different versions of the RE dynamic region demonstrate that unlike RI deletion, removal of short RE- $\Delta \mathrm{RE}_{S}\left(\Delta^{654} \mathrm{Ser}-\mathrm{Gly}{ }^{673}\right)$ did not per se 
rescue F508del-CFTR processing. Nevertheless, $\triangle \mathrm{RE}_{\mathrm{S}}$ dramatically stabilized the immature form of F508del-CFTR (see Figure 6). In fact, our pulse-chase experiments show that the immature form of $\triangle \mathrm{RE}_{\mathrm{S}}$-F508del-CFTR exhibits a turnover rate which is $\sim 2$-fold lower than that of wt-CFTR. Of note, when Aleksandrov et al. removed the dynamic region RI from F508del-CFTR they found a dramatic increase in the channel thermostability, even augmented for higher temperatures [13]. Interestingly however, while $\mathrm{RE}_{\mathrm{S}}$ removal from $\Delta \mathrm{RI}_{\mathrm{L}}$-F508del-CFTR did not significantly affect processing $(71 \% \mathrm{vs}$. $78 \%$ for $\Delta \mathrm{RE}_{\mathrm{S}}-\Delta \mathrm{RI}_{\mathrm{L}}-\mathrm{F} 508 \mathrm{del}-\mathrm{CFTR}$ and $\Delta \mathrm{RI}_{\mathrm{L}}-\mathrm{F} 508 \mathrm{del}-\mathrm{CFTR}$, respectively, in Table 1, it further reduced its function from 92 to $78 \%$ ( $\Delta \mathrm{RI}_{\mathrm{L}}$-F508del-CFTR vs. $\Delta \mathrm{RE}_{\mathrm{S}}-\Delta \mathrm{RI}_{\mathrm{L}}$-F508del-CFTR, respectively, Figure $\left.5 \mathrm{C}\right)$.

The latter findings on function of $\Delta \mathrm{RE}_{\mathrm{S}}-\mathrm{wt}-\mathrm{CFTR}$ and $\Delta \mathrm{RE}_{\mathrm{S}}-\Delta \mathrm{RI}_{\mathrm{L}}-\mathrm{F} 508 \mathrm{del}-\mathrm{CFTR}$ were somewhat surprising since the RE was previously described to impede putative NBD1:NBD2 dimerization that is required for channel gating [20]. Accordingly, an increase in CFTR activity would be expected upon $\triangle R E_{S}$ removal, which is actually the opposite of what we observe for wt-CFTR. Besides the fact that those authors studied a different RE version $\left({ }^{639} \mathrm{Asp}-\mathrm{Ser}^{670}\right)$, this discrepancy could derive from structural constraints and lack of flexibility of the 'single polypeptide' protein that we used, vs. their 'split' CFTR channels (2 'halves' of 633 and 668 amino acid residues) for which channel function was indistinguishable from wt-CFTR [20]. Moreover, since RD phosphorylation is required for channel gating [24,25], the reduced function of $\triangle \mathrm{RE}_{\mathrm{S}}$-wt-CFTR may also result from absence of ${ }^{660} \mathrm{Ser}$ and ${ }^{670}$ Ser $[17,21]$. Although the RD contains more than ten PKA phospho-sites and no individual one is essential, phosphorylation of increasing numbers of sites enables progressively greater channel activity [26].

Removal of a longer RE version $\left(\Delta \mathrm{RE}_{\mathrm{L}}: \Delta^{647} \mathrm{Cys}-\mathrm{Ser}^{678}\right)$ was without effect on F508del-CFTR processing and significantly reduced that of $w t-C F T R$ to $88 \%$. Such differential impact on wt- and F508del-CFTR is consistent with the conformational heterogeneity between these two proteins lacking both RI and RE [27].

Removal of RI short version, $\mathrm{RI}_{\mathrm{S}}\left(\Delta^{412} \mathrm{Ala}-\mathrm{Leu}^{428}\right)$ significantly reduced wt-CFTR processing to less than half of its normal levels, while not rescuing F508del-CFTR processing, thus, being essential for CFTR proper folding. This is in contrast to removal of long $\mathrm{RI}\left(\Delta \mathrm{RI}_{\mathrm{L}}\right)$ which led to $78 \%$ processing F508del-CFTR, as reported [13] but without impact on wt-CFTR. These data indicate that those 8/7 amino acid residues at the $\mathrm{N}$-term/ C-term of $\mathrm{RI}_{\mathrm{S}}$ (and absent in $\mathrm{RI}_{\mathrm{L}}$ ) impair the folding efficiency and processing of both wt- and F508del-CFTR. Despite the difficulty in speculating how those amino acid residues can specifically impact of F508del-CFTR folding and processing, the fact that structurally $\mathrm{RI}_{\mathrm{S}}$ is strictly the region described as destructured in the crystal structure [19] and $\mathrm{RI}_{\mathrm{L}}$ includes some flanking residues restricting its mobility may explain the observed difference.

Indeed, the recently published cryo-EM structures of human CFTR $[22,28,29]$ indicate that $\mathrm{RI}_{\mathrm{S}}$ is structurally disordered. Consistently, the RI loop in NBD1 is described in the zebrafish CFTR cryo-structure $[30,31]$ to contribute to the amorphous density that is observed between NBD1 and the elbow helix of TMD2 [32]. Our data for the RI are consistent with these findings.

From a structural point of view, models of the full-length CFTR protein suggest that the two regions (RI and RE) behave differently. The distances between the extremities of the deleted parts are indeed different $\left(\Delta \mathrm{RI}_{\mathrm{S}}=18 \AA, \Delta \mathrm{RI}_{\mathrm{L}}=10 \AA\right.$ versus $\left.\Delta \mathrm{RE}_{\mathrm{S}}=27 \AA, \Delta R E_{\mathrm{L}}=30 \AA\right)$. The longer distances observed for $\triangle R E$ imply a substantial reorganization of the C-terminal parts of NBD1 and NBD2, which precludes a clear understanding of what might happen upon these deletions. In contrast, the lower distances observed for $\Delta \mathrm{RI}$ (near a possible minimum of $6 \AA$ ) allows a better simulation of the possible structural behavior of these constructs. Indeed, preliminary molecular dynamics simulations of the $\Delta \mathrm{RI}_{\mathrm{L}}$-F508del-CFTR suggested that in this case, part of the RD is reorganized and partially takes space left by the $\triangle$ RI deletion, thereby substituting it for tight contacts with NBD2. Meanwhile, following a probable allosteric effect [13,18,32], contact of NBD1-F508 with ICL4 residue L1077 (a likely essential contact for channel opening), is nearly completely restored by I507. Such a feature is not observed in the F508del-CFTR model [33]. 
G550E

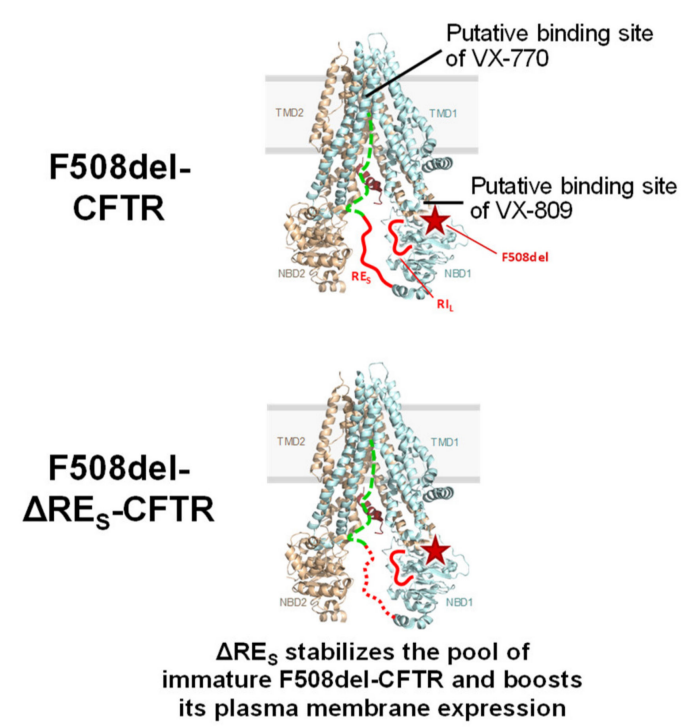

mmature F508del-CFTR and boost
F508del$\Delta \mathrm{RI}_{\mathrm{L}}-\mathrm{CFTR}$

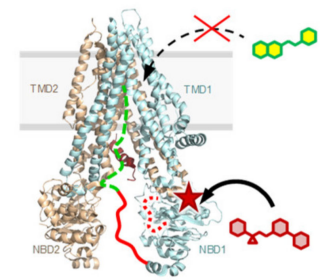

$\Delta \mathrm{RI}_{\mathrm{L}}$ synergises with $\mathrm{VX}-809$ (but not with revertants) to rescue $\triangle R_{\mathrm{s}}-\mathrm{F} 508 \mathrm{del}-\mathrm{CFTR}$ processing, but decreases by half the VX-770 stimulated current (vs Gen/Fsk)

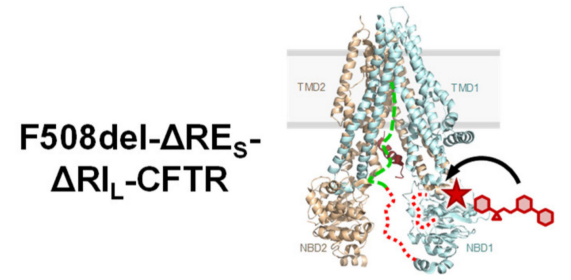

$\Delta \mathrm{RI}_{\mathrm{L}}-\Delta R \mathrm{E}_{\mathrm{s}}-\mathrm{F}$ 508del-CFTR levels at the $P M$ are equivalent to those of wt-CFTR and VX-809 causes even further rescue
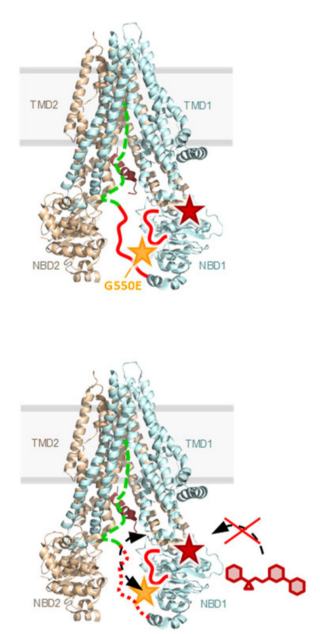

$\triangle R E_{S}$ interferes with the positive impact of VX-809 on F508del-CFTR with the revertants (R1070W, G550E)
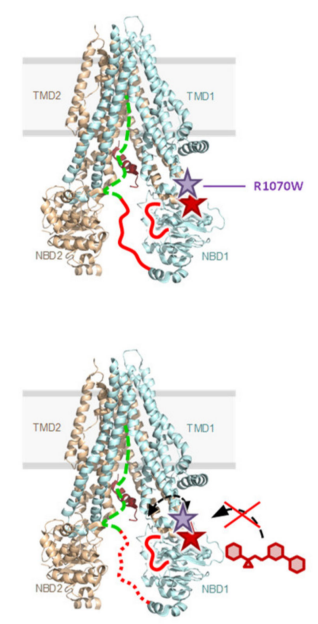

R1070W

A most significant effect of $\Delta R I_{L}$ is the almost abolition of VX-770 stimulated current of G550EF508del-CFTR (vs Gen/Fsk)

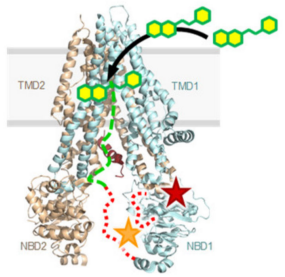

$\Delta \mathrm{RE}_{\mathrm{s}}$ increases F508delCFTR currents in response to VX-770 when combined with $\Delta \mathrm{RI}_{\mathrm{L}}$

Figure 6. Summary of the most relevant results observed in the present study. CFTR structure used is the one by Liu F et al. [22] and putative bind sites of VX-809 and VX-770 shown are the NBD1:MSD2 (ICL4) interface and to the MSDs (although it is not defined the exact binding site), as described by Farinha et al. [5] and by Jih and Hwang [23], respectively. $\mathrm{RI}_{\mathrm{L}}$ and $\mathrm{RE}_{\mathrm{S}}$ are shown as red lines, the $\mathrm{R}$ region a dashed green line.

\subsection{Effect of VX-809 on F508del-CFTR Variants Lacking RE and RI}

As for rescue of CFTR variants lacking RE and RI by CFTR modulators, VX-809 restored the processing of both $\Delta \mathrm{RE}_{\mathrm{S}}-\Delta \mathrm{RI}_{\mathrm{L}}$ - and $\Delta \mathrm{RI}_{\mathrm{L}}$-F508del-CFTR variants equally well and to wt-CFTR levels (from $71-78 \%$ to $92-96 \%$. These data suggest a strong synergistic effect between VX-809 and $\Delta \mathrm{RI}_{\mathrm{L}}$ to rescue $\Delta \mathrm{RE}_{\mathrm{S}}$-F508del-CFTR processing and thus some possible interference of the regulatory insertion with VX-809 binding to F508del-CFTR (see Figure 6). Indeed, although previous studies [34] suggested 
putative binding of VX-809 to NBD1:MSD2 (ICL4) interface (see Figure 6) or to TMD1 [35], they also suggested possibility of further F508del-CFTR correction at distinct conformational sites, so data shown here suggest that VX-809 may also bind to the regulatory insertion.

Interestingly, the processing defect of $\Delta \mathrm{RI}_{\mathrm{S}}$-wt-CFTR (but not of $\Delta \mathrm{RI}_{\mathrm{S}}$-F508del-CFTR) was rescuable by VX-809 to $90 \%$, indicating that the amino acid stretches of the $\mathrm{RI}_{\mathrm{L}}$ that remain present in $\mathrm{RI}_{\mathrm{S}}$ do not affect the rescue of $\triangle \mathrm{RI}_{\mathrm{S}}$-wt-CFTR but preclude rescue of F508del-CFTR by VX-809.

We also tested the impact of removing helix 9 (H9) which precedes the RE $\left({ }^{635} \mathrm{Gln}-\mathrm{Gly}^{646}\right)$, just after $\mathrm{H} 8\left({ }^{630} \mathrm{Phe}-\mathrm{Leu}^{634}\right)$, both helices proposed to interact with the NBD1:NBD2 heterodimer interface by folding onto the NBD1 $\beta$-subdomain [21,27] as well as to bind ICL4 near Phe508. When H9 is present, $\triangle \mathrm{RE}_{\mathrm{L}}$-wt-CFTR processing appears to be favored by VX-809, suggesting some synergy of this small molecule with $\mathrm{H} 9$ helix to correct the conformational defect(s) caused by $\triangle \mathrm{RE}_{\mathrm{L}}$ on wt-CFTR. These data are in contrast to $\Delta \mathrm{H} 9-\mathrm{F} 508 \mathrm{del}-\mathrm{CFTR}$ which exhibits $0 \%$ processing with or without $\Delta \mathrm{RE}_{\mathrm{L}}$ and no rescue by VX-809. We can assume that $\mathrm{H} 9$ also contacts $\mathrm{RE}$, at least in some states, such as when the RE adopts different conformations as previously suggested [19].

Most strikingly, and in contrast to its effect on processing, was the effect of $\Delta \mathrm{RI}_{\mathrm{L}}$ on the VX-770 stimulated currents which were decreased by almost half vs those stimulated by Gen/Fsk $(92 \%$ to $58 \%$ ). These data seem to indicate that the absence of the regulatory insertion could impair (and its presence favor) binding of VX-770 to CFTR (see Figure 6). Surprisingly, the removal of RES from $\triangle \mathrm{RI}_{\mathrm{L}}$-F508del-CFTR could correct this defect and restore the maximal function by VX-770 (see Figure 6). Indeed, under VX-770, $\triangle \mathrm{RE}_{\mathrm{S}}-\Delta \mathrm{RI}_{\mathrm{L}}$-F508del-CFTR exhibited significantly higher activity (87\%) than $\Delta \mathrm{RI}_{\mathrm{L}}$-F508del-CFTR (58\%). In contrast, removal of $\mathrm{RE}_{\mathrm{S}}$ from wt-CFTR significantly reduced its functionin comparison with that of wt-CFTR (down to 70\%) but had no effect on processing. Our study does not address the mechanism coupling the RI to F508del-CFTR pharmacological rescue, namely, why is the RI essential for rescue by VX-770 or why is it inhibitory for rescue by VX-809. Nevertheless, insight may be obtained from experimental structural data. In a recent cryo-EM structure of full-length human CFTR [29] VX-770 was found to bind in a transmembrane location coinciding with a hinge involved in gating. The authors suggested that populating this binding pocket may stabilize the intermolecular rotation opening the CFTR channel upon ATP binding at the NBD1-NBD2 interface. Given that the RI is located near the NBD interface and ATP binding site [29] we may speculate that RI deletion hampers NBD dimerization and, therefore, rescue by VX-770. Regarding VX-809, we may speculate that the RI interferes with drug binding.

\subsection{Impact of F508del-Revertants on CFTR Variants Lacking RE and RI}

Another goal of the present study was to assess how presence of the F508del-CFTR revertants G550E and R1070W [5,10,11] influence variants without RE and RI. Remarkably, our data show that the presence of either of these revertants did not affect $\triangle R E_{S}-F 508$ del-CFTR processing, but both of them further increased processing (but not function) of $\Delta \mathrm{RI}_{\mathrm{L}}-\mathrm{F} 508 \mathrm{del}-\mathrm{CFTR}$ to almost levels of wt-CFTR: 92-96\% (G550E) and 71-76\% (R1070W).

In contrast, removal of $\mathrm{RI}_{\mathrm{S}}$ from either of these F508del-CFTR revertants completely abolished their processing, emphasizing how important the different residues between $\mathrm{RI}_{\mathrm{S}}$ and $\mathrm{RI}_{\mathrm{L}}$ are for F508del-CFTR conformers partially rescued by the revertants. We can speculate that $\mathrm{RI}_{S}$ removal from F508del-CFTR has an effect on folding equivalent to that of either G550E or R1070W.

Interestingly, regarding wt-CFTR processing, G550E (at the NBD1:NBD2 dimer interface) partially recovered the negative effect caused by $\mathrm{RI}_{\mathrm{S}}$ removal, thus further suggesting that this revertant and the destructured region of RI may be allosterically coupled, since they do not plausibly interact (Figure S5). On the contrary, R1070W (at the NBD1:ICL4 interface), negatively affected processing of wt-CFTR (to $69 \%$ ) and of $\Delta \mathrm{RI}_{\mathrm{S}}$-wt-CFTR (from $44 \%$ to $11 \%$ ), while not affecting $\Delta \mathrm{RI}_{\mathrm{L}}$-wt-CFTR. R1070W rescues F508del-CFTR because ${ }^{1070} \mathrm{Trp}$ fills the gap created by deletion of residue ${ }^{508} \mathrm{Phe}$ [36]. It is not surprising that it perturbs CFTR folding due to clashing of ${ }^{508} \mathrm{Phe}$ and ${ }^{1070} \mathrm{Trp}$ residues. It is nevertheless, curious that R1070W affect more the processing $\Delta \mathrm{RI}_{\mathrm{S}}-\mathrm{wt}-\mathrm{CFTR}$ than wt-CFTR, to levels of F508del-CFTR, 
suggesting that both changes affect the same region of the molecule. Rescue of both R1070W-wt-CFTR and R1070W- $\Delta \mathrm{RI}_{\mathrm{S}}-\mathrm{wt}-\mathrm{CFTR}$ by VX-809 further supports this concept.

The most striking effect of $\Delta \mathrm{RI}_{\mathrm{L}}$ however, was the almost complete abolition of VX-770-stimulated current of G550E-F508del-CFTR to levels even lower than those observed for $\Delta \mathrm{RI}_{\mathrm{L}}-\mathrm{F} 508 \mathrm{del}-\mathrm{CFTR}$ (see above). It was suggested that VX-770 binds directly to CFTR to the MSDs (although it is not defined the exact binding site), in phosphorylation-dependent but ATP-independent manner and away from the canonical catalytic site $[23,37]$. Both RD and RI were suggested by Eckford and colleagues as putative binding regions for VX-770 [37]. Nevertheless, the pharmacological effect of VX-770 remains robust in the absence of the RD [23] and here we demonstrate that it does indeed require RI since VX-770 is unable to stimulate either $\Delta \mathrm{RI}_{\mathrm{L}}$-F508del-CFTR or $\Delta \mathrm{RI}_{\mathrm{L}}-\mathrm{G} 550 \mathrm{E}-\mathrm{F} 508 \mathrm{del}-\mathrm{CFTR}$ (see Figure 6). We can speculate that removal of $\mathrm{RI}_{\mathrm{L}}$ increases the chances of $\mathrm{VX}-770$ blocking the pore. In contrast, this would not occur for genistein, which has been proposed to bind to the NBDs interface [38], thus probably explaining why it does not cause this effect.

\section{Materials and Methods}

\subsection{CFTR Variants, Cells, and Culture Conditions}

Several CFTR deletion variants were produced by site-directed mutagenesis corresponding

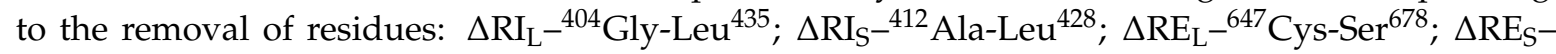

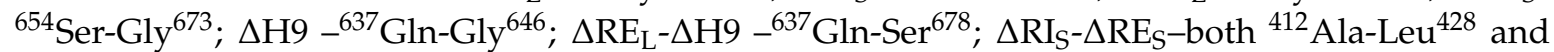
${ }^{654}$ Ser-Gly ${ }^{673}$; and $\Delta \mathrm{RI}_{\mathrm{L}}-\Delta \mathrm{RE}_{\mathrm{S}}{ }^{4}{ }^{404} \mathrm{Gly}-\mathrm{Leu}^{435}$ and ${ }^{654} \mathrm{Ser}-\mathrm{Gly}{ }^{673}$. All the constructs were produced using full length wt-CFTR and F508del-CFTR.

BHK cells lines expressing $\Delta \mathrm{RI}_{\mathrm{L}}-, \Delta \mathrm{RI}_{\mathrm{L}}$-F508del-, $\Delta \mathrm{RI}_{\mathrm{L}}$-G550E-, $\Delta \mathrm{RI}_{\mathrm{L}}-\mathrm{G} 550 \mathrm{E}-\mathrm{F} 508 \mathrm{del}, \Delta \mathrm{RI}_{\mathrm{L}}-\mathrm{R} 1070 \mathrm{~W}-$, $\Delta \mathrm{RI}_{\mathrm{L}}-\mathrm{R} 1070 \mathrm{~W}-\mathrm{F} 508 \mathrm{del}, \Delta \mathrm{RI}_{\mathrm{S}^{-}}, \Delta \mathrm{RI}_{\mathrm{S}}$-F508del, $\Delta \mathrm{RI}_{\mathrm{S}}$-G550E-, $\Delta \mathrm{RI}_{\mathrm{S}}$-G550E-F508del, $\Delta \mathrm{RI}_{\mathrm{S}}-\mathrm{R} 1070 \mathrm{~W}-$,

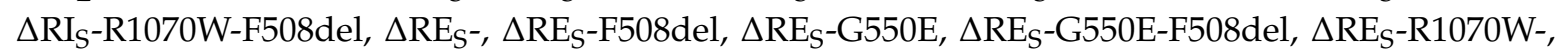
$\Delta \mathrm{RE}_{\mathrm{S}}-\mathrm{R} 1070 \mathrm{~W}-\mathrm{F} 508 \mathrm{del}, \Delta \mathrm{RE}_{\mathrm{L}^{-}}, \Delta \mathrm{RE}_{\mathrm{L}}-\mathrm{F} 508 \mathrm{del}, \Delta \mathrm{RI}_{\mathrm{S}}-\Delta \mathrm{RE}-, \Delta \mathrm{RI}_{\mathrm{S}}-\Delta \mathrm{RE}_{\mathrm{S}}-\mathrm{F}$ 08del, $\Delta \mathrm{RI}_{\mathrm{L}}-\Delta \mathrm{RE}-, \Delta \mathrm{RI}_{\mathrm{L}^{-}}$ $\Delta \mathrm{RE}_{\mathrm{S}}$-F508del, $\Delta \mathrm{H} 9-, \Delta \mathrm{H} 9-\mathrm{F} 508 \mathrm{del}, \Delta \mathrm{RE}_{\mathrm{L}}-\Delta \mathrm{H} 9-$, and $\Delta \mathrm{RE}_{\mathrm{L}}-\Delta \mathrm{H} 9-\mathrm{F} 508 \mathrm{del}-\mathrm{CFTR}$ were produced and cultured as previously described ${ }^{11}$. Cells were cultured in DMEM/F-12 medium containing $5 \%(v / v)$ Fetal bovine serum (FBS) and $500 \mu \mathrm{M}$ of methotrexate. For some experiments, cells were incubated with $3 \mu \mathrm{M}$ VX-809 or with the equivalent concentration of Dimethyl sulfoxide (DMSO, Control) for $48 \mathrm{~h}$ at $37^{\circ} \mathrm{C}$.

\subsection{Western Blot}

To study the effect of removal of regulatory extension (RE) and or regulatory insertion (RI) in combination with genetic revertants and VX-809, cells were incubated for $48 \mathrm{~h}$ at $37^{\circ} \mathrm{C}$ with $3 \mu \mathrm{M}$ VX-809. After incubation, cells were lysed, and extracts analyzed by Western blot (WB) using the anti-CFTR 596 antibody $(\mathrm{Ab})$ or anti-calnexin $\mathrm{Ab}$ as a loading control. Score corresponds to the percentage of band $C$ to total CFTR (bands $B+C$ ) as normalized to the same ratio in samples from wt-CFTR expressing cells. Blot images were acquired using BioRad ChemiDoc XRC+ imaging system and band intensities were measured using Image Lab analysis software.

\subsection{Pulse-Chase and Immunoprecipitation}

BHK cells lines stably expressing CFTR variants were starved for $30 \mathrm{~min}$ in methionine-free $\alpha$-modified Eagle's medium or minimal essential medium and then pulsed for $30 \mathrm{~min}$ in the same medium supplemented with $100 \mu \mathrm{Ci} / \mathrm{mL}\left[{ }^{35} \mathrm{~S}\right]$ methionine. After chasing for $0,0.5,1,1.5,2$, and $3 \mathrm{~h}$ in a-modified Eagle's medium with $8 \%(v / v)$ fetal bovine serum and $1 \mathrm{mM}$ non-radioactive methionine, cells were lysed in $1 \mathrm{~mL}$ of Radioimmunoprecipitation assay (RIPA) buffer [1\% ( $w / v)$ deoxycholic acid, $1 \%(v / v)$ Triton X-100, 0.1\% (w/v) Sodium dodecyl sulfate (SDS), $50 \mathrm{mM}$ Tris, $\mathrm{pH} 7.4$, and $150 \mathrm{mM}$ $\mathrm{NaCl}$. The immunoprecipitation (IP) was carried out using the anti-CFTR 596 antibody in independent experiments and Protein G-agarose or Protein A-Sepharose beads. Immunoprecipitated proteins were 
eluted from the beads with sample buffer for $1 \mathrm{~h}$ at room temperature and then electrophoretically separated on $7 \%(w / v)$ polyacrylamide gels. Gels were pre-fixed in methanol/acetic acid $(30: 10, v / v)$, washed in water and, for fluorography, soaked in $1 \mathrm{M}$ sodium salicylate for $60 \mathrm{~min}$. After drying at $80^{\circ} \mathrm{C}$ for $2 \mathrm{~h}$, gels were exposed to $\mathrm{X}$-ray films and further analyzed and quantified by densitometry.

\subsection{Iodide Efflux}

CFTR-mediated iodide effluxes were measured at room temperature using the cAMP agonist forskolin (Fsk $10 \mu \mathrm{M})$ and the CFTR potentiator genistein (Gen, $50 \mu \mathrm{M})$ or VX-770 $(10 \mu \mathrm{M})$ and Gen $(50 \mu \mathrm{M})$.

\subsection{Biochemical Determination of the Plasma Membrane Levels of CFTR}

To determine plasma membrane levels of CFTR protein, we performed cell surface biotinylation in BHK cells cultured on permeable growth supports or tissue culture plates using cell membrane impermeable EZ-Link ${ }^{\mathrm{TM}}$ Sulfo-NHS-SS-Biotin, followed by cell lysis in buffer containing 25 mM HEPES, $\mathrm{pH} 8.0,1 \%(v / v)$ Triton, $10 \%$ glycerol $(v / v)$, and Complete Protease Inhibitor Mixture, as described previously $[39,40]$. Biotinylated proteins were isolated by streptavidin-agarose beads, eluted into SDS-sample buffer, and separated by $7.5 \%(w / v)$ SDS-PAGE.

\subsection{Multiple Sequence Alignment}

Sequences for NBD domains of $A B C$ transporters were obtained from Uniprot [41] (human and mouse CFTR) or the PDB [42] (1B0U, 1L2T, 1G29, 1G6H, and 1JJ7). Alignments were performed with Jalview [43] using the T-Coffee [44] algorithm with default parameters.

\subsection{Data and Statistical Analyses}

The data and statistical analyses used in this study comply with the recommendations on experimental design and data analysis in pharmacology [45]. Quantitative results are shown as mean \pm SEM of $n$ observations. To compare two sets of data, Student's $t$-test was used, and differences considered to be significant for $p$-values $\leq 0.05$. In Western blotting (Figures 1-3), pulse chase (Figure 4 and Figure S2); cell surface biotinylation (Figure 4) and in iodide efflux studies (Table 1, Figure 5, Figures S3 and S4), $\mathrm{n}$ represents the number of experiments performed with distinct cell cultures on different days, being thus biological replicates.

\subsection{Reagents}

All reagents used here were of the highest purity grade available. Forskolin and genistein were from Sigma-Aldrich (St. Louis, MI, USA); VX-809 and VX-770 were acquired from Selleck Chemicals (Houston, TX, USA). CFTR was detected with the mouse anti-CFTR monoclonal 596Ab, which recognizes a region of NBD2 (1204-1211) from CFFT-Cystic Fibrosis Foundation Therapeutics CFF Therapeutics [46] and calnexin with rabbit polyclonal anti-calnexin Ab SPA-860, from Stressgen Biotechnologies Corporation (Victoria, BC, Canada). Other specific reagents included: X-ray films (Kodak, supplied by Sigma-Aldrich); Complete Protease Inhibitor Mixture from Roche Applied Science (Penzberg, Germany); EZ-Link ${ }^{\mathrm{TM}}$ Sulfo-NHS-SS-Biotin from Pierce Chemical Company (Rockford, IL, USA).

\section{Conclusions}

Overall, our data show that while the presence of the regulatory insertion (RI) seems to preclude full rescue of F508del-CFTR processing by VX-809, this region appears essential to rescue its function by VX-770, thus suggesting some contradictory role in rescue of F508del-CFTR by these two modulators (Figure 7). Nevertheless, this negative impact of removing RI on VX-770-stimulated currents on F508del-CFTR can be compensated by deletion of the regulatory extension which also 
leads to the stabilization of this mutant. We thus propose that, despite both these regions being conformationally dynamic, RI precludes F508del-CFTR processing while RE affects mostly its stability and channel opening.

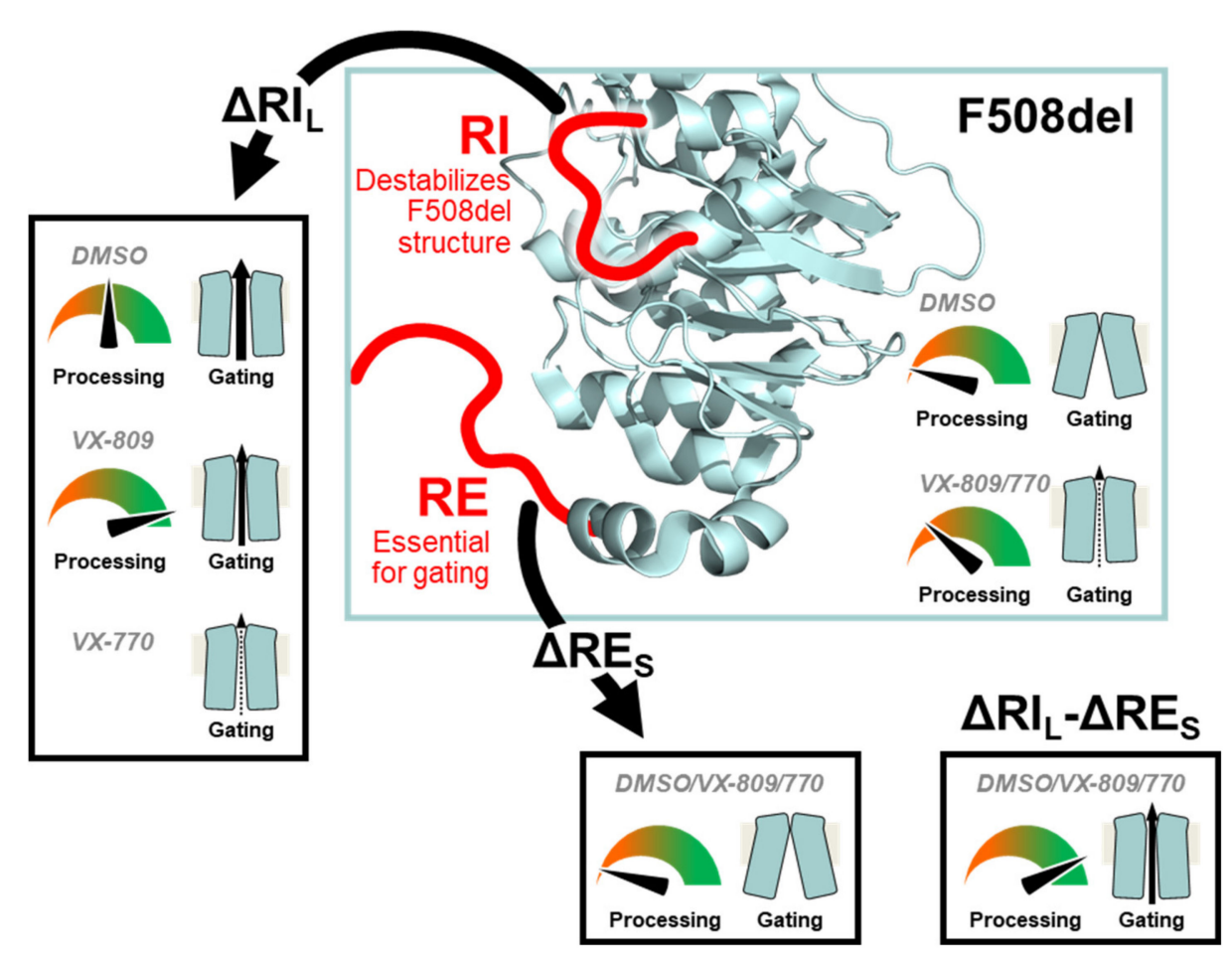

Figure 7. Model for the modulation of F508del processing and gating by $\mathrm{RI}_{\mathrm{L}}$ and $\mathrm{RE}_{\mathrm{S}}$. Our data suggest that the RI destabilizes the F508del-CFTR structure. Therefore, deleting the RI $\left(\triangle R I_{L}\right)$ rescues F508del-CFTR processing and gating. However, the RI is essential for F508del-CFTR rescuing by VX-770. The $R E$ is essential for gating and removal of its shorter version $\left(\triangle R E_{S}\right)$ generates an F508del-CFTR variant which cannot be rescued pharmacologically. Simultaneous removal of both regions $\left(\Delta \mathrm{RI}_{\mathrm{L}}-\Delta \mathrm{RE}_{\mathrm{S}}\right)$ rescues F508del-CFTR processing and gating. The CFTR structure from Liu F et al. [22] depicts the overall NBD1 structure and the location of the RI and RE.

Supplementary Materials: Supplementary materials can be found at http://www.mdpi.com/1422-0067/21/12/ 4524/s1, Figure S1. ABC transporters alignment. Sequence alignment of NBD sequences from ABC transporters. Sequences correspond to NBD domains with experimentally determined 3D structures: human CFTR (hNBD1) and Tap1, mouse CFTR (mNBD1), HisP from S. typhimurium, MJ0796 and MJ1267 from M. jannaschii and Malk from T. litoralis. Only RI and RE regions are shown. Numbering is the one of human CFTR. The localization of the RI, RE and H9 are shown as arrows and shades. Blue and pink regions respectively highlight structured and unstructured stretches in human NBD1 crystal structures [19], Figure S2. Turnover and processing of wt-CFTR, alone or in cis with $\triangle \mathrm{RE}_{\mathrm{S}}$ and $\Delta \mathrm{RI}_{\mathrm{S}}$. (A) BHK cells expressing wt, $\Delta \mathrm{RE}_{\mathrm{S}}$ and $\Delta \mathrm{RI}_{\mathrm{S}}$ were labelled with ${ }^{35} \mathrm{~S}$-methionine for $30 \mathrm{~min}$ and then chased for the indicated times $(0,0.5,1,2,3 \mathrm{~h})$ before lysis. IP was performed with the anti-CFTR 596 antibody. After electrophoresis and fluorography, images were analysed by densitometry. (B) Turnover of immature (band B) CFTR for different CFTR variants is shown as the percentage of immature protein at a given time point of chase $(\mathrm{P})$ relative to the amount at $t=0\left(\mathrm{P}_{0}\right)$. $(\mathrm{C})$ Efficiency of processing of band $\mathrm{B}$ into band $C$ is shown as the percentage of band $C$ at a given time of chase relative to the amount of band $B$ at $t=0$. Data are mean \pm SEM at each point $(n=3)$, Figure S3. Functional characterization of the $\Delta R_{S}$ and $\Delta \mathrm{RI}_{\mathrm{L}} \mathrm{variants}$ of F508del-CFTR with and without VX-809 or VX-770 treatments. (A-L) Iodide efflux from BHK cells stably expressing $\Delta \mathrm{RE}_{\mathrm{S}}, \Delta \mathrm{RI}_{\mathrm{S}}$ - and $\Delta \mathrm{RI}_{\mathrm{L}}-\mathrm{F} 508$ del-CFTR variants. Cells were incubated either with $3 \mu \mathrm{M} V \mathrm{VX}-809$ (E-H) or equivalent concentration of DMSO (control) for $48 \mathrm{~h}$ at $37^{\circ} \mathrm{C}$ (A-D; I-L) and for the assay stimulated with Fsk/Gen (A-H) or Fsk/VX-770 (I-L), as indicated above the bar and for the period indicated by the bar, Figure S4. Functional characterization of the $\triangle \mathrm{RE}_{\mathrm{S}}$ and $\triangle \mathrm{RI}_{\mathrm{L}}$ variants of wt-CFTR. (A-C) Iodide efflux from BHK cells stably expressing $\triangle \mathrm{RE}_{\mathrm{S}}$ - or $\triangle \mathrm{RI}$-wt-CFTR alone (A) or jointly with revertants R1070W and G550E (B, C). Cells were stimulated during the assay with Fsk/Gen (A, B) or with Fsk/VX-770 (C), as indicated above the bar and for the period indicated by the bar. (D) Graph summarizing data from the iodide efflux peak magnitude generated by different BHK cells stably expressing the various CFTR variants. Data are shown as a percentage of wt-CFTR activity and as mean \pm SEM. (n) indicates number of independent experiments. "\#" indicates significantly different from the 
wt-CFTR stimulated with Fsk/Gen $(p<0.05)$. (E) Quantification of the iodide efflux data, showing peak response (time and value) and percentage of wt-CFTR activity, Figure S5. Relative localization of the RI and G550 in CFTR. The figure shows the structure of full length CFTR bound to ATP and VX-770 [34] viewed from the intracellular side, highlighting the NBDs and rendering secondary structure elements and protein surface. The RI is shown in shades of red: light red highlights the stretch which is resolved in the cryo-EM structure [34] $\left({ }^{404} \mathrm{Gly}^{-P h e}{ }^{409}\right.$ ) and dark red highlights the ${ }^{410} \mathrm{Glu}^{-L_{e}}{ }^{435}$ stretch, which is not resolved. This stretch is shown as modelled by Serohijos et al. [4], after alignment to NBD1. Other elements are colored as in Figure 1A. (A) Overall structure. (B) Cut through the NBDs. Interaction between the RI and the G550 site is unlikely given their separation and the buried localization of G550.

Author Contributions: Conceptualization, I.U., A.C.d.P., and M.D.A.; data curation, I.U., A.C.d.P., H.M.B., and M.D.A.; formal analysis, I.U., H.M.B., and C.M.F.; funding acquisition, M.D.A.; Investigation, I.U., A.C.d.P., S.A., M.J.L., V.F., H.M.B., and C.M.F.; methodology, I.U., A.C.d.P., H.M.B., and M.D.A.; project administration, I.U.; Supervision, I.U. and M.D.A.; validation, I.U.; writing original draft, I.U., H.M.B., and M.D.A.; review and editing I.U., H.M.B. and M.D.A. All authors have read and agreed to the published version of the manuscript.

Funding: Work supported by grants UIDB/04046/2020 and UIDP/04046/2020 centre grants (to BioISI) and research grant (to M.D.A.): "iDrugCF" (FCT/02/SAICT/2017/28800), both from FCT/MCTES Portugal '. I.U., A.C.P. and V.F. were recipients of SFRH/BD/69180/2010, SFRH/BD/17475/2004, SFRH/BD/87478/2012 PhD fellowships and H.M.B. of SFRH/BPD/93017/2013 post-doctoral fellowship (FCT, Portugal), respectively.

Acknowledgments: Authors acknowledge I Callebaut, B Hoffmann, and J-P Mornon for the revision of the manuscript and for the helpful comments.

Conflicts of Interest: The authors declare no conflict of interest. The funders had no role in the design of the study; in the collection, analyses, or interpretation of data; in the writing of the manuscript, or in the decision to publish the results.

\section{References}

1. Riordan, J.R. CFTR Function and Prospects for Therapy. Annu. Rev. Biochem. 2008, 77, 701-726. [CrossRef] [PubMed]

2. Sheppard, D.N.; Welsh, M.J. Structure and Function of the CFTR Chloride Channel. Physiol. Rev. 1999, 79, S23-S45. [CrossRef] [PubMed]

3. Kanelis, V.; Hudson, R.P.; Thibodeau, P.H.; Thomas, P.J.; Forman-Kay, J.D. NMR Evidence for Differential Phosphorylation-Dependent Interactions in WT and DeltaF508 CFTR. EMBO J. 2010, 29, 263-277. [CrossRef] [PubMed]

4. Serohijos, A.W.R.; Hegedus, T.; Aleksandrov, A.A.; He, L.; Cui, L.; Dokholyan, N.V.; Riordan, J.R. Phenylalanine-508 Mediates a Cytoplasmic-Membrane Domain Contact in the CFTR 3D Structure Crucial to Assembly and Channel Function. Proc. Natl. Acad. Sci. USA 2008, 105, 3256-3261. [CrossRef]

5. Farinha, C.M.; King-Underwood, J.; Sousa, M.; Correia, A.R.; Henriques, B.J.; Roxo-Rosa, M.; Da Paula, A.C.; Williams, J.; Hirst, S.; Gomes, C.M.; et al. Revertants, Low Temperature, and Correctors Reveal the Mechanism of F508del-CFTR Rescue by VX-809 and Suggest Multiple Agents for Full Correction. Chem. Biol. 2013, 20, 943-955. [CrossRef]

6. He, L.; Aleksandrov, A.A.; Serohijos, A.W.R.; Hegedus, T.; Aleksandrov, L.A.; Cui, L.; Dokholyan, N.V.; Riordan, J.R. Multiple Membrane-Cytoplasmic Domain Contacts in the Cystic Fibrosis Transmembrane Conductance Regulator (CFTR) Mediate Regulation of Channel Gating. J. Biol. Chem. 2008, 283, 26383-26390. [CrossRef]

7. Denning, G.M.; Anderson, M.P.; Amara, J.F.; Marshall, J.; Smith, A.E.; Welsh, M.J. Processing of Mutant Cystic Fibrosis Transmembrane Conductance Regulator Is Temperature-Sensitive. Nature 1992, 358, 761-764. [CrossRef]

8. Teem, J.L.; Berger, H.A.; Ostedgaard, L.S.; Rich, D.P.; Tsui, L.C.; Welsh, M.J. Identification of Revertants for the Cystic Fibrosis Delta F508 Mutation Using STE6-CFTR Chimeras in Yeast. Cell 1993, 73, 335-346. [CrossRef]

9. Teem, J.L.; Carson, M.R.; Welsh, M.J. Mutation of R555 in CFTR-Delta F508 Enhances Function and Partially Corrects Defective Processing. Recept. Channels 1996, 4, 63-72.

10. DeCarvalho, A.C.V.; Gansheroff, L.J.; Teem, J.L. Mutations in the Nucleotide Binding Domain 1 Signature Motif Region Rescue Processing and Functional Defects of Cystic Fibrosis Transmembrane Conductance Regulator Delta F508. J. Biol. Chem. 2002, 277, 35896-35905. [CrossRef] 
11. Roxo-Rosa, M.; Xu, Z.; Schmidt, A.; Neto, M.; Cai, Z.; Soares, C.M.; Sheppard, D.N.; Amaral, M.D. Revertant Mutants G550E and 4RK Rescue Cystic Fibrosis Mutants in the First Nucleotide-Binding Domain of CFTR by Different Mechanisms. Proc. Natl. Acad. Sci. USA 2006, 103, 17891-17896. [CrossRef] [PubMed]

12. Loo, T.W.; Bartlett, M.C.; Clarke, D.M. The V510D Suppressor Mutation Stabilizes DeltaF508-CFTR at the Cell Surface. Biochemistry 2010, 49, 6352-6357. [CrossRef]

13. Aleksandrov, A.A.; Kota, P.; Aleksandrov, L.A.; He, L.; Jensen, T.; Cui, L.; Gentzsch, M.; Dokholyan, N.V.; Riordan, J.R. Regulatory Insertion Removal Restores Maturation, Stability and Function of DeltaF508 CFTR. J. Mol. Biol. 2010, 401, 194-210. [CrossRef] [PubMed]

14. Van Goor, F.; Hadida, S.; Grootenhuis, P.D.J.; Burton, B.; Stack, J.H.; Straley, K.S.; Decker, C.J.; Miller, M.; McCartney, J.; Olson, E.R.; et al. Correction of the F508del-CFTR Protein Processing Defect in Vitro by the Investigational Drug VX-809. Proc. Natl. Acad. Sci. USA 2011, 108, 18843-18848. [CrossRef] [PubMed]

15. Lewis, H.A.; Buchanan, S.G.; Burley, S.K.; Conners, K.; Dickey, M.; Dorwart, M.; Fowler, R.; Gao, X.; Guggino, W.B.; Hendrickson, W.A.; et al. Structure of Nucleotide-Binding Domain 1 of the Cystic Fibrosis Transmembrane Conductance Regulator. EMBO J. 2004, 23, 282-293. [CrossRef] [PubMed]

16. Dahan, D.; Evagelidis, A.; Hanrahan, J.W.; Hinkson, D.A.; Jia, Y.; Luo, J.; Zhu, T. Regulation of the CFTR Channel by Phosphorylation. Pflüg. Arch. Eur. J. Physiol. 2001, 443, S92-S96. [CrossRef]

17. Pasyk, S.; Molinski, S.; Ahmadi, S.; Ramjeesingh, M.; Huan, L.-J.; Chin, S.; Du, K.; Yeger, H.; Taylor, P.; Moran, M.F.; et al. The Major Cystic Fibrosis Causing Mutation Exhibits Defective Propensity for Phosphorylation. Proteomics 2015, 15, 447-461. [CrossRef] [PubMed]

18. Dawson, J.E.; Farber, P.J.; Forman-Kay, J.D. Allosteric Coupling between the Intracellular Coupling Helix 4 and Regulatory Sites of the First Nucleotide-Binding Domain of CFTR. PLoS ONE 2013, 8, e74347. [CrossRef]

19. Lewis, H.A.; Zhao, X.; Wang, C.; Sauder, J.M.; Rooney, I.; Noland, B.W.; Lorimer, D.; Kearins, M.C.; Conners, K.; Condon, B.; et al. Impact of the DeltaF508 Mutation in First Nucleotide-Binding Domain of Human Cystic Fibrosis Transmembrane Conductance Regulator on Domain Folding and Structure. J. Biol. Chem. 2005, 280, 1346-1353. [CrossRef]

20. Csanády, L.; Chan, K.W.; Nairn, A.C.; Gadsby, D.C. Functional Roles of Nonconserved Structural Segments in CFTR's NH2-Terminal Nucleotide Binding Domain. J. Gen. Physiol. 2005, 125, 43-55. [CrossRef]

21. Bozoky, Z.; Krzeminski, M.; Muhandiram, R.; Birtley, J.R.; Al-Zahrani, A.; Thomas, P.J.; Frizzell, R.A.; Ford, R.C.; Forman-Kay, J.D. Regulatory R Region of the CFTR Chloride Channel Is a Dynamic Integrator of Phospho-Dependent Intra- and Intermolecular Interactions. Proc. Natl. Acad. Sci. USA 2013, 110, E4427-E4436. [CrossRef] [PubMed]

22. Liu, F.; Zhang, Z.; Csanády, L.; Gadsby, D.C.; Chen, J. Molecular Structure of the Human CFTR Ion Channel. Cell 2017, 169, 85-95.e8. [CrossRef] [PubMed]

23. Jih, K.-Y.; Hwang, T.-C. Vx-770 Potentiates CFTR Function by Promoting Decoupling between the Gating Cycle and ATP Hydrolysis Cycle. Proc. Natl. Acad. Sci. USA 2013, 110, 4404-4409. [CrossRef] [PubMed]

24. Chappe, V.; Irvine, T.; Liao, J.; Evagelidis, A.; Hanrahan, J.W. Phosphorylation of CFTR by PKA Promotes Binding of the Regulatory Domain. EMBO J. 2005, 24, 2730-2740. [CrossRef]

25. Mense, M.; Vergani, P.; White, D.M.; Altberg, G.; Nairn, A.C.; Gadsby, D.C. In Vivo Phosphorylation of CFTR Promotes Formation of a Nucleotide-Binding Domain Heterodimer. EMBO J. 2006, 25, 4728-4739. [CrossRef]

26. Hegedus, T.; Serohijos, A.W.R.; Dokholyan, N.V.; He, L.; Riordan, J.R. Computational Studies Reveal Phosphorylation-Dependent Changes in the Unstructured R Domain of CFTR. J. Mol. Biol. 2008, 378, 1052-1063. [CrossRef]

27. Hudson, R.P.; Chong, P.A.; Protasevich, I.I.; Vernon, R.; Noy, E.; Bihler, H.; An, J.L.; Kalid, O.; Sela-Culang, I.; Mense, M.; et al. Conformational Changes Relevant to Channel Activity and Folding within the First Nucleotide Binding Domain of the Cystic Fibrosis Transmembrane Conductance Regulator. J. Biol. Chem. 2012, 287, 28480-28494. [CrossRef]

28. Zhang, Z.; Liu, F.; Chen, J. Molecular Structure of the ATP-Bound, Phosphorylated Human CFTR. Proc. Natl. Acad. Sci. USA 2018, 115, 12757-12762. [CrossRef]

29. Liu, F.; Zhang, Z.; Levit, A.; Levring, J.; Touhara, K.K.; Shoichet, B.K.; Chen, J. Structural Identification of a Hotspot on CFTR for Potentiation. Science 2019, 364, 1184-1188. [CrossRef]

30. Zhang, Z.; Chen, J. Atomic Structure of the Cystic Fibrosis Transmembrane Conductance Regulator. Cell 2016, 167, 1586-1597.e9. [CrossRef] 
31. Zhang, Z.; Liu, F.; Chen, J. Conformational Changes of CFTR upon Phosphorylation and ATP Binding. Cell 2017, 170, 483-491.e8. [CrossRef] [PubMed]

32. Aleksandrov, A.A.; Kota, P.; Cui, L.; Jensen, T.; Alekseev, A.E.; Reyes, S.; He, L.; Gentzsch, M.; Aleksandrov, L.A.; Dokholyan, N.V.; et al. Allosteric Modulation Balances Thermodynamic Stability and Restores Function of $\triangle$ F508 CFTR. J. Mol. Biol. 2012, 419, 41-60. [CrossRef]

33. Hoffmann, B.; Callebaut, I.; Mornon, J.-P.; (Sorbonne University, Paris, France). Personal communication, 2016.

34. Hudson, R.P.; Dawson, J.E.; Chong, P.A.; Yang, Z.; Millen, L.; Thomas, P.J.; Brouillette, C.G.; Forman-Kay, J.D. Direct Binding of the Corrector VX-809 to Human CFTR NBD1: Evidence of an Allosteric Coupling between the Binding Site and the NBD1:CL4 Interface. Mol. Pharmacol. 2017, 92, 124-135. [CrossRef] [PubMed]

35. Ren, H.Y.; Grove, D.E.; De La Rosa, O.; Houck, S.A.; Sopha, P.; Van Goor, F.; Hoffman, B.J.; Cyr, D.M. VX-809 Corrects Folding Defects in Cystic Fibrosis Transmembrane Conductance Regulator Protein through Action on Membrane-Spanning Domain 1. Mol. Biol. Cell 2013, 24, 3016-3024. [CrossRef] [PubMed]

36. Thibodeau, P.H.; Richardson, J.M.; Wang, W.; Millen, L.; Watson, J.; Mendoza, J.L.; Du, K.; Fischman, S.; Senderowitz, H.; Lukacs, G.L.; et al. The Cystic Fibrosis-Causing Mutation DeltaF508 Affects Multiple Steps in Cystic Fibrosis Transmembrane Conductance Regulator Biogenesis. J. Biol. Chem. 2010, 285, 35825-35835. [CrossRef]

37. Eckford, P.D.W.; Li, C.; Ramjeesingh, M.; Bear, C.E. Cystic Fibrosis Transmembrane Conductance Regulator (CFTR) Potentiator VX-770 (Ivacaftor) Opens the Defective Channel Gate of Mutant CFTR in a Phosphorylation-Dependent but ATP-Independent Manner. J. Biol. Chem. 2012, 287, 36639-36649. [CrossRef]

38. Wang, F.; Zeltwanger, S.; Yang, I.C.-H.; Nairn, A.C.; Hwang, T.-C. Actions of Genistein on Cystic Fibrosis Transmembrane Conductance Regulator Channel Gating. J. Gen. Physiol. 1998, 111, 477-490. [CrossRef]

39. Moyer, B.D.; Loffing, J.; Schwiebert, E.M.; Loffing-Cueni, D.; Halpin, P.A.; Karlson, K.H.; Ismailov, I.I.; Guggino, W.B.; Langford, G.M.; Stanton, B.A. Membrane Trafficking of the Cystic Fibrosis Gene Product, Cystic Fibrosis Transmembrane Conductance Regulator, Tagged with Green Fluorescent Protein in Madin-Darby Canine Kidney Cells. J. Biol. Chem. 1998, 273, 21759-21768. [CrossRef]

40. Swiatecka-Urban, A.; Duhaime, M.; Coutermarsh, B.; Karlson, K.H.; Collawn, J.; Milewski, M.; Cutting, G.R.; Guggino, W.B.; Langford, G.; Stanton, B.A. PDZ Domain Interaction Controls the Endocytic Recycling of the Cystic Fibrosis Transmembrane Conductance Regulator. J. Biol. Chem. 2002, 277, 40099-40105. [CrossRef]

41. UniProt Consortium. UniProt: A Hub for Protein Information. Nucleic Acids Res. 2015, 43, D204-D212. [CrossRef]

42. Berman, H.M.; Westbrook, J.; Feng, Z.; Gilliland, G.; Bhat, T.N.; Weissig, H.; Shindyalov, I.N.; Bourne, P.E. The Protein Data Bank. Nucleic Acids Res. 2000, 28, 235-242. [CrossRef] [PubMed]

43. Waterhouse, A.M.; Procter, J.B.; Martin, D.M.A.; Clamp, M.; Barton, G.J. Jalview Version 2-a Multiple Sequence Alignment Editor and Analysis Workbench. Bioinforma. Oxf. Engl. 2009, 25, 1189-1191. [CrossRef] [PubMed]

44. Notredame, C.; Higgins, D.G.; Heringa, J. T-Coffee: A Novel Method for Fast and Accurate Multiple Sequence Alignment. J. Mol. Biol. 2000, 302, 205-217. [CrossRef]

45. Curtis, M.J.; Bond, R.A.; Spina, D.; Ahluwalia, A.; Alexander, S.P.A.; Giembycz, M.A.; Gilchrist, A.; Hoyer, D.; Insel, P.A.; Izzo, A.A.; et al. Experimental Design and Analysis and Their Reporting: New Guidance for Publication in BJP. Br. J. Pharmacol. 2015, 172, 3461-3471. [CrossRef] [PubMed]

46. Cui, L.; Aleksandrov, L.; Chang, X.-B.; Hou, Y.-X.; He, L.; Hegedus, T.; Gentzsch, M.; Aleksandrov, A.; Balch, W.E.; Riordan, J.R. Domain Interdependence in the Biosynthetic Assembly of CFTR. J. Mol. Biol. 2007, 365, 981-994. [CrossRef]

(C) 2020 by the authors. Licensee MDPI, Basel, Switzerland. This article is an open access article distributed under the terms and conditions of the Creative Commons Attribution (CC BY) license (http://creativecommons.org/licenses/by/4.0/). 